Algebraic $8 \mathcal{G}$ Geometric $\mathcal{T}$ opology

Volume 5 (2005) 577-613

Published: 30 June 2005

ATG

\title{
Surfaces in the complex projective plane and their mapping class groups
}

\author{
Susumu Hirose
}

\begin{abstract}
An orientation preserving diffeomorphism over a surface embedded in a 4-manifold is called extendable, if this diffeomorphism is a restriction of an orientation preserving diffeomorphism on this 4-manifold. In this paper, we investigate conditions for extendability of diffeomorphisms over surfaces in the complex projective plane.
\end{abstract}

AMS Classification 57Q45; 57N05, 20F38

Keywords Knotted surface, plane curve, mapping class group, spin mapping class group

Dedicated to Professor Yukio Matsumoto for his 60th birthday

\section{Introduction}

There are deformations of embedded surfaces in 4-manifolds which induce isotopically non-trivial diffeomorphisms on surfaces. We introduce two typical examples.

For the first example, we consider a deformation of an annulus embedded in $S^{3} \times[-1,1]$ so that, under this deformation, the boundary of this annulus is fixed. Let $S^{1} \times[0,1]$ be an annulus embedded in $S^{3} \times\{0\} \subset S^{3} \times[-1,1]$, and $t: S^{3} \times[-1,1] \rightarrow[-1,1]$ a projection to the second factor. We deform $S^{1} \times[0,1]$ as in Figure 1. First, we isotope $S^{1} \times[0,1]$ in $S^{3}$ from (1) to (3). Next, we isotope $S^{1} \times[0,1]$ so that outside of the annulus $A$ of (3) $t=0$, and inside $t>0$. Then we isotope $S^{1} \times[0,1]$ inside $A$ so that, when we push $A$ down to $S^{3} \times\{0\}, S^{1} \times[0,1]$ is as in (4). Finally, we isotope $S^{1} \times[0,1]$ in $S^{3}$ from (4) to (6). The composition of these deformations induce a square of Dehn twist about the core circle $S^{1} \times\left\{\frac{1}{2}\right\}$ of $S^{1} \times[0,1]$.

For the second example, we consider a deformation of a non-singular plane curve of degree 3. A torus is defined as a quotient of the complex plane by a lattice $\mathbb{Z}+\mathbb{Z} \sqrt{-1}$. We embed this torus into the complex projective plane 


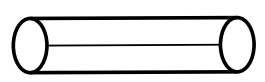

(1)

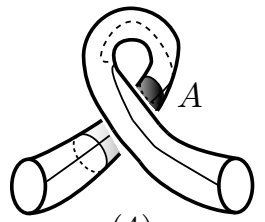

(4)

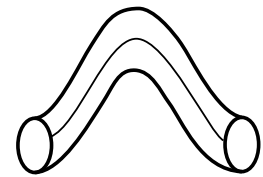

$(2)$

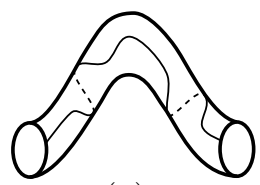

(5)

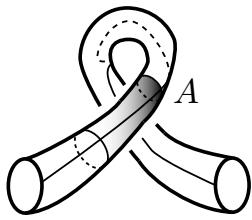

(3)

Figure 1

by using the Weierstrass $\wp$ function associated to this lattice, then the image of this embedding is a non-singular plane curve of degree 3 . We deform this lattice, $\mathbb{Z}+\mathbb{Z}(\sqrt{-1}+t)$, where $0 \leq t \leq 1$ is a parameter of this deformation. Then the embedding is deformed isotopically and, finally (when $t=1$ ), brought back to the original position. This deformation induces a Dehn twist on the non-singular plane curve of degree 3 .

In this paper, we investigate a topological meaning of the above phenomena.

We settle a general formulation. Let $M$ be a simply connected compact oriented smooth 4-manifold (possibly with boundary) and $F$ be a compact oriented smooth 2-manifold (possibly with boundary) embedded in $M$. We call the pair $(M, F)$ a knotted surface. In particular, if $F$ is characteristic, that is to say, $F \cdot X \equiv X \cdot X \bmod 2$ for any $X \in H_{2}(M, \mathbb{Z})$, then we call this pair $(M, F)$ a knotted characteristic surface. An orientation preserving diffeomorphism $\psi$ over $F$ is extendable if there is an orientation preserving diffeomorphism $\Psi$ over $M$ such that $\left.\Psi\right|_{F}=\psi$. In general, for an oriented manifold $A$ and its submanifold $B$, we denote

$$
\operatorname{Diff}_{+}(A, \text { fix } B)=\left\{\begin{array}{l|l}
\psi & \begin{array}{l}
\text { an orientation preserving diffeomorphism over } A \\
\text { such that }\left.\psi\right|_{B}=i d_{B}
\end{array}
\end{array}\right\} .
$$

If $B=\phi$, we denote this group by $\operatorname{Diff}_{+}(M)$. The group $\pi_{0}\left(\operatorname{Diff}_{+}(F\right.$, fix $\left.\partial F)\right)$ is called the mapping class group of $F$ and denoted by $\mathcal{M}_{F}$. If $F$ is a closed oriented surface of genus $g$, this group is denoted by $\mathcal{M}_{g}$. We define

$$
\mathcal{E}(M, F)=\left\{\psi \in \mathcal{M}_{F} \mid \psi \text { is extendable }\right\} .
$$


This is a subgroup of $\mathcal{M}_{F}$ and is a central object of this paper.

In the case where $M=S^{4}$, there are several works on this group. Let $\left(S^{4}, \Sigma_{g}\right)$ be the genus $g$ trivial knotted surface in $S^{4}$. When $g=1$, Montesinos 19. investigated $\mathcal{E}\left(S^{4}, \Sigma_{1}\right)$, and when $g \geq 2$, the author [1] investigated $\mathcal{E}\left(S^{4}, \Sigma_{g}\right)$. Let $\left(S^{3}, k\right)$ be a knot in $S^{3}$ and $\left(S^{4}, S(k)\right)$ (resp. $\left.\left(S^{4}, \tilde{S}(k)\right)\right)$ the spun (resp. the twisted spun) of $\left(S^{3}, k\right)$. When $\left(S^{3}, k\right)$ is a torus knot, Iwase 13 investigated $\mathcal{E}\left(S^{4}, S(k)\right)$ and $\mathcal{E}\left(S^{4}, \tilde{S}(k)\right)$, and when $\left(S^{3}, k\right)$ is an arbitrary knot, the author 10] investigated these groups.

In this paper, we investigate the case where $M$ is the complex projective plane $\mathbb{C P}^{2}$. In $₫ 3$, we treat the case where $\left(\mathbb{C P}^{2}, \Sigma_{g}\right)$ is a standard embedding of $\Sigma_{g}$. In 4 , we treat the case where $\left(\mathbb{C P}^{2}, F\right)$ is a non-singular plane curve. From $\$ 5$ to the end of this paper, we treat the case where $\left(\mathbb{C P}^{2}, F\right)$ is a connected sum of a non-singular plane curve of degree 3 and a trivial embedding.

\section{Preliminary: A Hopf band on the boundary of the 4-ball}

A link $L$ in $S^{3}$ is called a fibered link if there is a map $\phi: S^{3} \backslash L \rightarrow S^{1}$ which is a fiber bundle projection. For each $t \in S^{1}, \phi^{-1}(t)=F$, which does not depend on $t$, is called the fiber of $L$. Since $\phi$ is a bundle projection, $S^{3} \backslash L$ is diffeomorphic to the quotient of $F \times[0,1]$ by an equivalence $(x, 0) \sim(h(x), 1)$ where $h$ is a diffeomorphism over $F$ and called the monodromy of $L$.

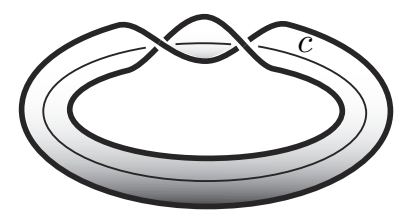

Positive Hopf band

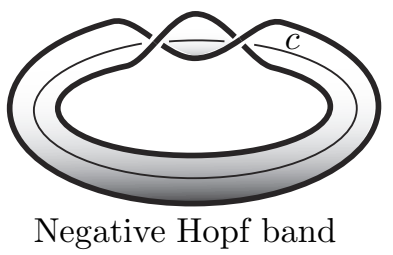

Figure 2

A Hopf band is an annulus embedded in $S^{3}$ as in Figure 2. In this picture, there are two types of Hopf bands. In this note, we treat both types of Hopf bands. The boundary of a Hopf band is called a Hopf link. The Hopf link is a fibered link whose fiber is the Hopf band and whose monodromy is a Dehn twist about the core circle of the Hopf band. Let $B$ be a Hopf band in $S^{3}$ which is a boundary of a 4-ball $D^{4}$. We push the interior of $B$ into the interior of $D^{4}$ and let $B^{\prime}$ be the annulus obtained by this deformation and let $c$ be the core circle of $B^{\prime}$. 
Proposition 2.1 The Dehn twist $T_{c}$ about $c$ is extendable, i.e. there is an element $T \in \operatorname{Diff}_{+}\left(D^{4}\right.$, fix $\left.\partial D^{4}\right)$ such that $\left.T\right|_{B^{\prime}}=T_{c}$.

Proof Since $\partial B$ is a fibered link, whose fiber is $B$ and whose monodromy is $T_{c}$, there is an orientation preserving diffeomorphism $\psi$ of $S^{3}$ such that $\left.\psi\right|_{B}=T_{c}$, and there is an isotopy $\psi_{t}(t \in[0,1])$ with $\psi_{0}=i d_{S^{3}}$ and $\psi_{1}=\psi$, which is defined by shifting fibers. Let $N\left(\partial D^{4}\right)$ be the regular neighborhood of $\partial D^{4}$ in $D^{4}$. We parametrize $N\left(\partial D^{4}\right)=S^{3} \times[0,2]$ so that $S^{3} \times\{0\}=\partial D^{4}$ and $B^{\prime}=\partial B \times[0,1] \cup B \times\{1\}$. Let $T$ be a diffeomorphism defined as follows

$$
\begin{aligned}
& \left.T\right|_{N\left(\partial D^{4}\right)}(x, t)= \begin{cases}\left(\psi_{t}(x), t\right) & 0 \leq t \leq 1 \\
\left(\psi_{2-t}(x), t\right) & 1 \leq t \leq 2\end{cases} \\
& \left.T\right|_{D^{4} \backslash N\left(\partial D^{4}\right)}=i d .
\end{aligned}
$$

This is the diffeomorphism which we need.

Remark 2.2 Let $\left(S^{4}, \Sigma_{g}\right)$ be the genus $g$ surface standardly embedded in $S^{4}$. In [1], the author showed that $T_{c_{4}} T_{c_{3}} T_{c_{4}}^{-1} \in \mathcal{E}\left(S^{4}, \Sigma_{g}\right)$ by using Montesinos' result [19, Theorem 5.3] ( $c_{3}$ and $c_{4}$ are as in Figure 17). We show this fact by using Proposition 2.1. The 4-sphere $S^{4}$ is constructed from two 4-balls $D_{+}^{4}$, $D_{-}^{4}$ with attaching along the boundary $S^{3}=\partial D_{+}^{4}=\partial D_{-}^{4}$. We parametrize the regular neighborhood $N\left(\partial D_{+}^{4}\right)=S^{3} \times[0,2]$ in $D_{+}^{4}$ so that $\partial D_{+}^{4}=S^{3} \times\{0\}$. The regular neighborhood $N$ of $T_{c_{4}}\left(c_{3}\right)$ in $\Sigma_{g}$ is a Hopf band in $S^{3} \subset S^{4}$. We push the interior of $N$ into the interior of $D_{+}^{4}$, then we get an annulus $N^{\prime}$ properly embedded in $D_{+}^{4}$. We may assume, by the above parametrization of $N\left(\partial D_{+}^{4}\right)$, $N^{\prime} \cap S^{3} \times\{t\}=\partial N \times\{t\}$ for $0 \leq t<2$ and $N^{\prime} \cap S^{3} \times\{2\}=N \times\{2\}$. We denote $D_{+}^{4} \backslash S^{3} \times[0,1)$ by $D^{\prime}$. By applying Proposition 2.1 to $\left(D^{\prime}, N^{\prime} \cap D^{\prime}\right)$, we show that there is an element $T \in \operatorname{Diff}_{+}\left(D^{\prime}\right.$, fix $\left.\partial D^{\prime}\right)$ such that $\left.T\right|_{N^{\prime} \cap D^{\prime}}=T_{c_{4}} T_{c_{3}} T_{c_{4}}^{-1}$. Therefore, we see $T_{c_{4}} T_{c_{3}} T_{c_{4}}^{-1} \in \mathcal{E}\left(S^{4}, \Sigma_{g}\right)$.

\section{Surfaces standardly embedded in the complex pro- jective plane}

For the free action of $\mathbb{C}^{*}=\mathbb{C} \backslash\{0\}$ on $\mathbb{C}^{3} \backslash\{(0,0,0)\}$ defined by $\lambda\left(z_{0}, z_{1}, z_{2}\right)=$ $\left(\lambda z_{0}, \lambda z_{1}, \lambda z_{2}\right)$, we take the quotient $\mathbb{C P}^{2}=\left(\mathbb{C}^{3} \backslash\{(0,0,0)\}\right) / \mathbb{C}^{*}$. This space $\mathbb{C P}^{2}$ is a closed oriented 4-manifold and called the complex projective plane. This 4-manifold $\mathbb{C P}^{2}$ is constructed from $D^{4}$ by attaching a 2-handle $h^{2}$ along the frame 1 trivial knot $K_{0}$ in $\partial D^{4}$, and attaching a 4-handle $h^{4}$. A 3dimensional handlebody $H_{g}$ is an oriented 3-manifold which is constructed from 
a 3-ball with attaching $g$ 1-handles. Any image of embeddings of $H_{g}$ into $\mathbb{C P}^{2}$ are isotopic each other. Therefore, $\left(\mathbb{C P}^{2}, \partial H_{g}\right)$ is unique. A surface standardly embedded in $\mathbb{C P}^{2}$ is $\left(\mathbb{C P}^{2}, \partial H_{g}\right)$. We obtain:

Theorem 3.1 For any $g, \mathcal{E}\left(\mathbb{C P}^{2}, \partial H_{g}\right)=\mathcal{M}_{g}$.

Proof Let $D^{4}$ be the 4-ball used to construct $\mathbb{C P}^{2}$ and $N\left(\partial D^{4}\right)$ be the regular neighborhood of $\partial D^{4}$ in $D^{4}$. We parametrize $N\left(\partial D^{4}\right)=S^{3} \times[0,-1]$, so that $S^{3} \times\{0\}=\partial D^{4}$ and, for $-1 \leq t<0, S^{3} \times\{t\}$ is in the interior of $D^{4}$. Since the image of embedding of $H_{g}$ in $\mathbb{C P}^{2}$ is unique up to isotopy, we assume that $H_{g}$ $\subset S^{3} \times\{-1\}$ and that each simple closed curve $c$ on $H_{g}$ which corresponds to Lickorish generator of mapping class group $\mathcal{M}_{g}$ is a trivial knot in $S^{3} \times\{-1\}$. The regular neighborhood $N(c)$ of $c$ on $\partial H_{g}$ is an annulus trivially embedded in $S^{3} \times\{-1\}$. At first, we deform $H_{g}$ in $S^{3} \times\{-1\}$ so that, if we forget the second factor $[0,-1], c \cup K_{0}$ becomes a Hopf link in $S^{3}$. We push $N(c)$ into $\partial\left(D^{4} \cup h^{2}\right)$, then $N(c)$ becomes a Hopf band in $\partial h^{4}$. By applying Proposition 2.1] we see that $T_{c}$ is extendable in $h^{4}$, and so in $\mathbb{C P}^{2}$.

\section{Non-singular plane curves}

We review here the topological description of non-singular plane curves by Akbulut and Kirby [1] (see also [6, 6.2.7]).
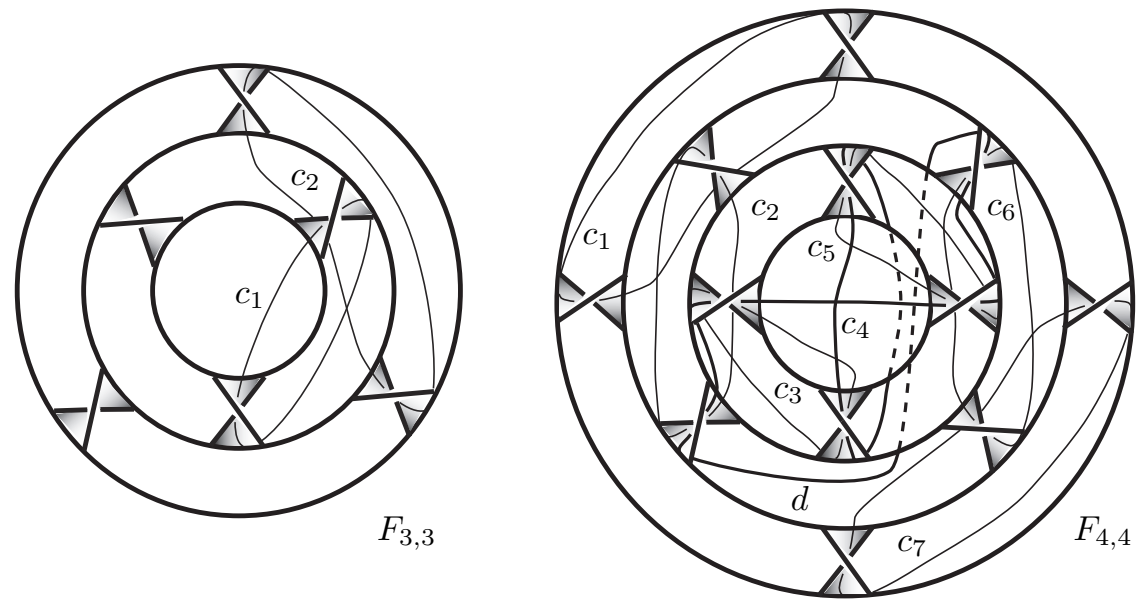

Figure 3 
An $(m, n)$-torus link $T_{m, n}$ is an oriented link in $S^{3}=\partial D^{4}$ consisting of $\operatorname{gcd}(m, n)$ oriented circles in the boundary of the tubular neighborhood $U$ of the trivial knot, representing $m \mu+n \lambda$ in $H_{1}(\partial U ; \mathbb{Z})$, where $\mu=$ [the meridian of the trivial knot] and $\lambda=$ [the longitude of the trivial knot]. There is a canonical Seifert surface $F_{m, n}$ for $T_{m, n}$, consisting of $n$-disks connected by $m(n-1)$ twisted bands as in Figure 3. As $K_{0}$, we take a trivial knot given by pushing $T_{1,0}$ into the complement of $U$ ( see the left hand side of Figure 4). From here, we consider only the case where $m=n=d$. As shown in the right hand side of Figure 4. $T_{d, d}$ becomes $d$ components trivial link in $\partial\left(D^{4} \cup h^{2}\right)$. Let $D_{d}$ be disjoint 2-disks in $\partial\left(D^{4} \cup h^{2}\right)$ which bound this trivial link.

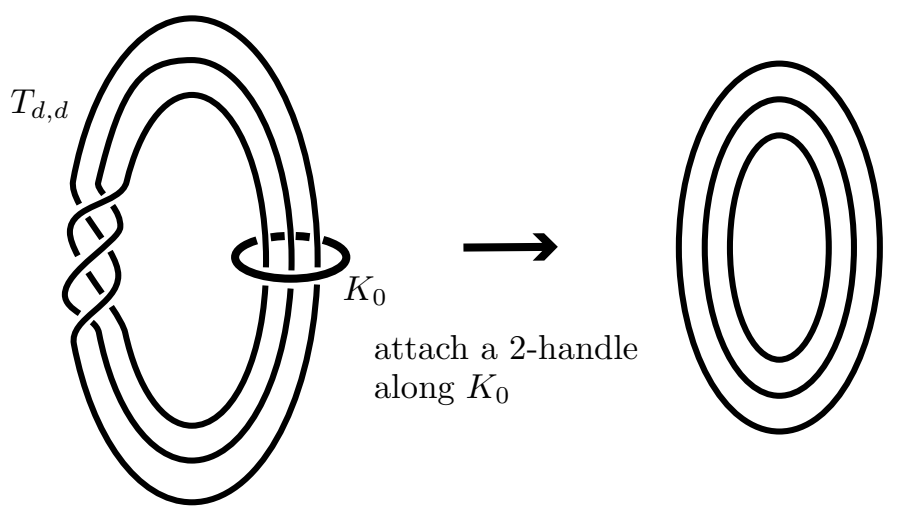

Figure 4

Let $K_{d}$ be a non-singular plane curve of degree $d$, then $K_{d}$ is a genus $\frac{(d-1)(d-2)}{2}$ closed oriented surface embedded in $\mathbb{C P}^{2}$. We remark that $K_{d}$ is unique up to isotopy, $K_{d}=\left\{[X: Y: Z] \in \mathbb{C P}^{2} \mid X^{d}+Y^{d}+Z^{d}=0\right\}$ and $\left[K_{d}\right]=d\left[\mathbb{C P}^{1}\right]$ $\in H_{2}\left(\mathbb{C P}^{2} ; \mathbb{Z}\right)$. Akbulut and Kirby showed:

Proposition 4.1 $K_{d}=F_{d, d} \cup D_{d}$.

Thus we obtain:

Theorem 4.2 When $d=3,4, \mathcal{E}\left(\mathbb{C P}^{2}, K_{d}\right)=\mathcal{M}_{g_{d}}$, where $g_{d}=\frac{(d-1)(d-2)}{2}$.

Proof When $d=3, K_{3}$ is homeomorphic to a 2-dimensional torus $T^{2}$. In $F_{3,3}$ (see Figure 3), each regular neighborhood of $c_{1}$ and $c_{2}$ is a Hopf band. Therefore, by Proposition 2.1. $T_{c_{1}}$ and $T_{c_{2}}$ are elements of $\mathcal{E}\left(\mathbb{C P}^{2}, K_{3}\right)$. On the other hand, $T_{c_{1}}$ and $T_{c_{2}}$ generate $\mathcal{M}_{1}$. Hence, $\mathcal{E}\left(\mathbb{C P}^{2}, K_{3}\right)=\mathcal{M}_{1}$. 


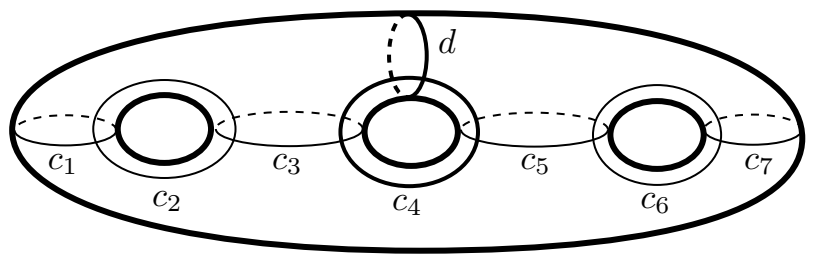

Figure 5

When $d=4$, we do the same as the above case. We remark that the Dehn twists about the simple closed curves in Figure 5 corresponding to the simple closed curves in $F_{4,4}$ (see Figure 3) with the same symbols generate the mapping class group of genus 3 surface [16].

When $d \geq 5, \mathcal{E}\left(\mathbb{C P}^{2}, K_{d}\right)$ is unknown. It is, however, not the case that $\mathcal{E}\left(\mathbb{C P}^{2}, K_{d}\right)=\mathcal{M}_{g_{d}}$, because, when $d$ is odd, $K_{d}$ is a characteristic surface, so the Rokhlin quadratic form on $H_{1}\left(K_{d} ; \mathbb{Z}_{2}\right)$ is well-defined (we review the definition of the Rokhlin quadratic form in the next section). By the definition of the Rokhlin quadratic form, if a diffeomorphism on $K_{d}$ is extendable to $\mathbb{C P}^{2}$, this diffeomorphism should preserve this form. Hence:

Theorem 4.3 When $d$ is an odd integer greater than or equal to $5, \mathcal{E}\left(\mathbb{C P}^{2}, K_{d}\right)$ is a proper subgroup of $\mathcal{M}_{g_{d}}$, where $g_{d}=\frac{(d-1)(d-2)}{2}$.

\section{Connected sum of the non-singular plane curve of degree 3 and trivial knotted surface}

We define knotted surfaces investigated from here to the end of this paper. The images of any embeddings of a 3-dimensional handlebody $H_{g}$ into $S^{4}$ are isotopic each other. We call this $\Sigma_{g}$-knot $\left(S^{4}, \partial H_{g}\right)$ a trivial $\Sigma_{g}$-knot, and this is denoted by $\left(S^{4}, \Sigma_{g}\right)$. Let $\left(\mathbb{C P}^{2}, K_{3}\right)$ be a nonsingular cubic plane curve. We define connected sum of $\left(\mathbb{C P}^{2}, K_{3}\right)$ and $\left(S^{4}, \Sigma_{g-1}\right)$ following the construction by Boyle [3] as follows. We choose points $p$ and $q$ on $K_{3}$ and $\Sigma_{g-1}$ respectively, and find small 4-balls $B_{1}$ and $B_{2}$ centered at $p$ and $q$ such that the pairs $\left(B_{1}, B_{1} \cap K_{3}\right)$ and $\left(B_{2}, B_{2} \cap \Sigma_{g-1}\right)$ are equivalent to the standard pair $\left(B^{4}, B^{2}\right)$. Now we glue the pairs $\left(S^{4} \backslash \operatorname{int}\left(B_{1}\right), K_{3} \backslash \operatorname{int}\left(B_{1}\right)\right)$ and $\left(\mathbb{C P}^{2} \backslash \operatorname{int}\left(B_{2}\right), \Sigma_{g-1} \backslash\right.$ $\left.\operatorname{int}\left(B_{2}\right)\right)$ together by an orientation-reversing diffeomorphism $f: \partial B_{1} \rightarrow \partial B_{2}$ such that $f\left(\partial B_{1} \cap K_{3}\right)=\partial B_{2} \cap \Sigma_{g-1}$. Since the connected sum of $\mathbb{C P}^{2}$ and $S^{4}$ is diffeomorphic to $\mathbb{C P}^{2}$, we get a surface in $\mathbb{C P}^{2}$ and denote this characteristic 
knotted surface by $\left(\mathbb{C P}^{2}, K_{3} \# \Sigma_{g-1}\right)$. From here to the end of this paper, we investigate on the group $\mathcal{E}\left(\mathbb{C P}^{2}, K_{3} \# \Sigma_{g-1}\right)$.

For a knotted characteristic surface $(M, F)$, where $M$ is a simply connected smooth closed oriented 4-manifold, we define a quadratic form (the Rokhlin quadratic form $) q_{F}: H_{1}\left(F ; \mathbb{Z}_{2}\right) \rightarrow \mathbb{Z}_{2}$ : Let $P$ be a compact surface embedded in $M$, with its boundary contained in $F$, normal to $F$ along its boundary, and its interior is transverse to $F$. Let $P^{\prime}$ be a surface transverse to $P$ obtained by sliding $P$ parallel to itself over $F$. Define $q_{F}([\partial P])=\#\left(\operatorname{int} P \cap\left(P^{\prime} \cup F\right)\right) \bmod 2$. This is a well-defined quadratic form with respect to the $\mathbb{Z}_{2}$-homology intersection form $(,)_{2}$ on $F$, i.e. for each pair of elements $x, y$ of $H_{1}\left(F ; \mathbb{Z}_{2}\right)$, $q_{F}(x+y)=q_{F}(x)+q_{F}(y)+(x, y)_{2}$. By the definition of the Rokhlin quadratic from $q_{F}$, if $\psi \in \operatorname{Diff}_{+}(F)$ is extendable, then $\psi$ preserves $q_{F}$, that is to say, $q_{F}\left(\psi_{*}(x)\right)=q_{F}(x)$ for any $x \in H_{1}\left(F ; \mathbb{Z}_{2}\right)$. We will show,

Theorem 5.1 For any $g \geq 2$,

$$
\mathcal{E}\left(\mathbb{C P}^{2}, K_{3} \# \Sigma_{g-1}\right)=\left\{\begin{array}{l|l}
\psi \in \mathcal{M}_{g} & \begin{array}{c}
q_{K_{3} \# \Sigma_{g-1}}\left(\psi_{*}(x)\right)=q_{K_{3} \# \Sigma_{g-1}}(x) \\
\text { for any } x \in H_{1}\left(K_{3} \# \Sigma_{g-1} ; \mathbb{Z}_{2}\right)
\end{array}
\end{array}\right\} .
$$

In 96 we investigate on a system of generators for the right hand side group in the equation of Theorem 5.1. In 97 , we show that each element of this system of generators is extendable.

\section{A finite set of generators for the odd spin mapping class group}

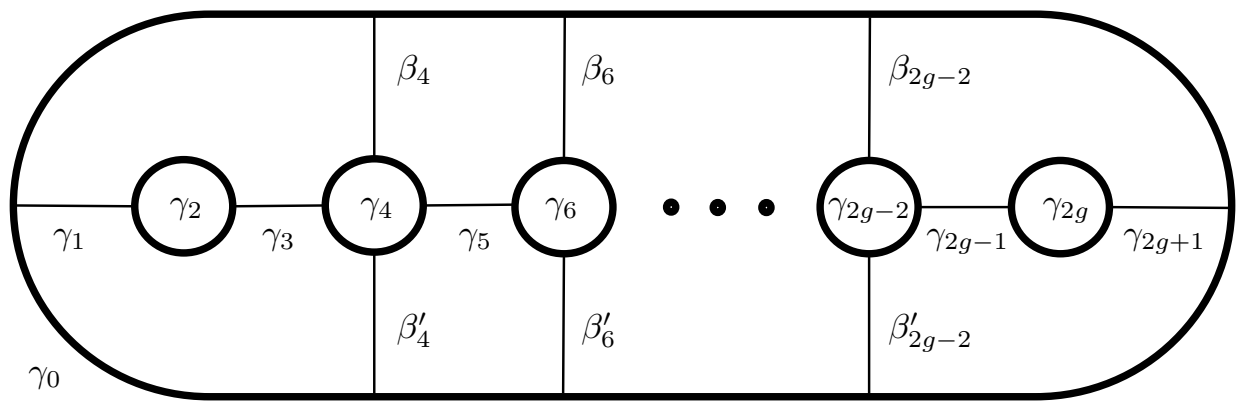

Figure 6 
We settle some notations. Let $P_{g}$ be a planar surface constructed from a 2-disk by removing $g$ copies of disjoint 2-disks. As indicated in Figure 6, we denote the boundary components of $P_{g}$ by $\gamma_{0}, \gamma_{2}, \ldots, \gamma_{2 g}$, and denote some properly embedded arcs of $P_{g}$ by $\gamma_{1}, \gamma_{3}, \ldots, \gamma_{2 g+1}, \beta_{4}, \ldots, \beta_{2 g-2}$ and $\beta_{4}^{\prime}, \ldots, \beta_{2 g-2}^{\prime}$. On $\partial\left(P_{g} \times[-1,1]\right)=\Sigma_{g}$, we define $c_{2 i-1}=\partial\left(\gamma_{2 i-1} \times[-1,1]\right)(1 \leq i \leq g+1)$, $b_{2 j}=\partial\left(\beta_{2 j} \times[-1,1]\right), b_{2 j}^{\prime}=\partial\left(\beta_{2 j}^{\prime} \times[-1,1]\right)(2 \leq j \leq g-1)$, and $c_{2 k}=\gamma_{2 k} \times\{0\}$ $(1 \leq k \leq g)$. In Figures 1 and 8 , these circles are illustrated and some of them are oriented.

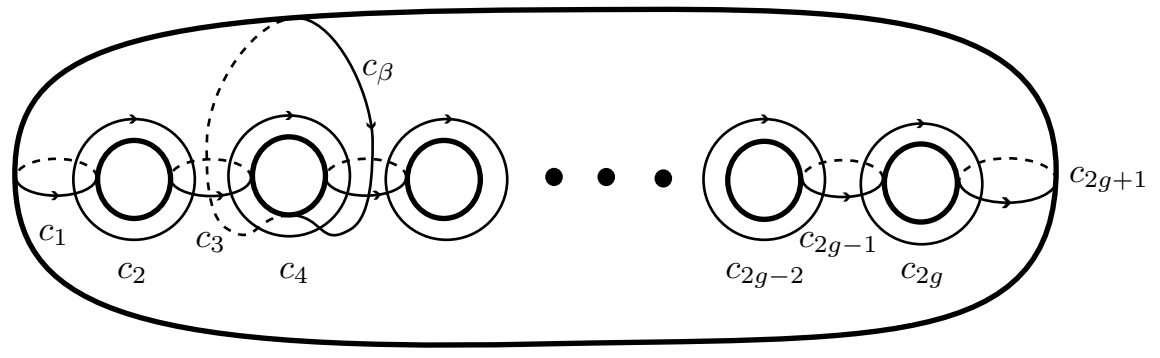

Figure 7

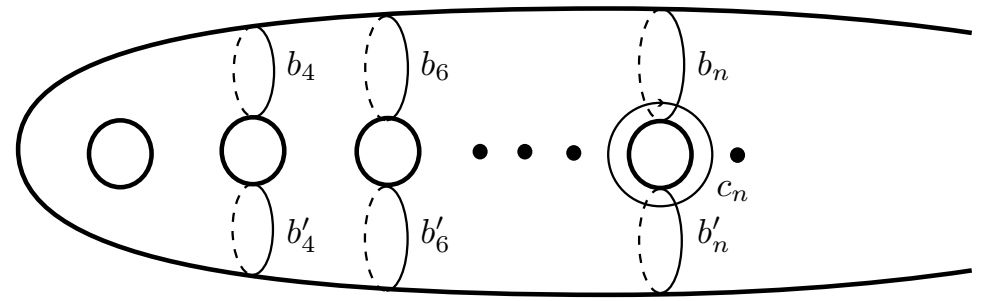

Figure 8

We set a basis of $H_{1}\left(\Sigma_{g} ; \mathbb{Z}\right)$ as in Figure 9, where $x_{1}=\left[c_{1}\right.$ with opposite orientation $], x_{i}=\left[b_{2 i}\right.$ with proper orientation $](2 \leq i \leq g-1), x_{g}=\left[c_{2 g+1}\right]$, and $y_{i}=\left[c_{2 i}\right.$ with opposite orientation $]$.

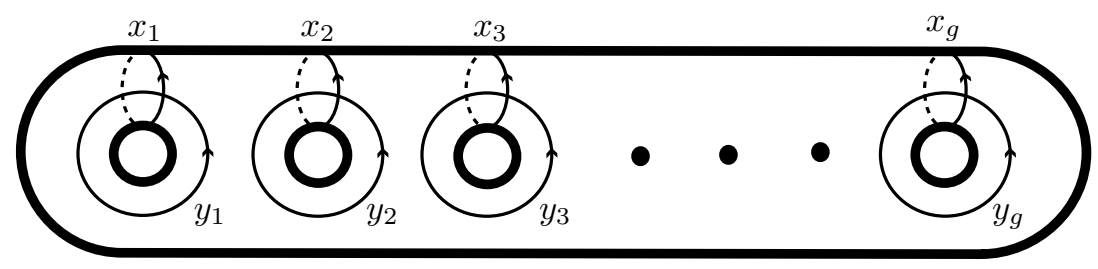

Figure 9 
A map $q: H_{1}\left(\Sigma_{g} ; \mathbb{Z}_{2}\right) \rightarrow \mathbb{Z}_{2}$ is called a quadratic form with respect to the $\mathbb{Z}_{2}$-homology intersection form $(,)_{2}$ on $\Sigma_{g}$ (for short, $\mathbb{Z}_{2}$-quadratic form on $\left.\Sigma_{g}\right)$ if $q(x+y)=q(x)+q(y)+(x, y)_{2}$, for each pair of elements $x, y$ of $H_{1}\left(\Sigma_{g} ; \mathbb{Z}_{2}\right)$. For the basis $\left\{x_{1}, y_{1}, \ldots, x_{g}, y_{g}\right\}$ introduced above, we define $\operatorname{Arf}(q)=\sum_{i=1}^{g} q\left(x_{i}\right) q\left(y_{i}\right)$. We call a $\mathbb{Z}_{2}$-quadratic form $q$ even quadratic from (resp. odd quadratic form) if $\operatorname{Arf}(q)=0$ (resp. $\operatorname{Arf}(q)=1$ ). We define

$$
\mathcal{S} \mathcal{P}_{g}[q]=\left\{\psi \in \mathcal{M}_{g} \mid q\left(\psi_{*}(x)\right)=q(x) \text { for any } x \in H_{1}\left(\Sigma_{g} ; \mathbb{Z}_{2}\right)\right\} .
$$

As is shown in [21], for two $\mathbb{Z}_{2}$-quadratic forms $q, q^{\prime}$ on $\Sigma_{g}$, if $\operatorname{Arf}(q)=$ $\operatorname{Arf}\left(q^{\prime}\right)$, then there is an element $\psi^{\prime} \in \mathcal{M}_{g}$ so that $q\left(\psi_{*}^{\prime}(x)\right)=q^{\prime}(x)$ for any $x$ $\in H_{1}\left(\Sigma_{g} ; \mathbb{Z}_{2}\right)$. Therefore, if $\operatorname{Arf}(q)=\operatorname{Arf}\left(q^{\prime}\right)$, then $\mathcal{S P}_{g}[q]$ and $\mathcal{S P}_{g}\left[q^{\prime}\right]$ are conjugate in $\mathcal{M}_{g}$. By the definition of $\mathbb{Z}_{2}$-quadratic from, values of a quadratic form is completely determined by its value for the basis of $H_{1}\left(\Sigma_{g} ; \mathbb{Z}_{2}\right)$. Let $q_{0}$ and $q_{1}$ be $\mathbb{Z}_{2}$-quadratic forms so that $q_{0}\left(x_{i}\right)=q_{0}\left(y_{i}\right)=0$ for $1 \leq i \leq g$, $q_{1}\left(x_{1}\right)=q_{1}\left(y_{1}\right)=1$ and $q_{1}\left(x_{j}\right)=q_{1}\left(y_{j}\right)=0$ for $2 \leq j \leq g$. Then $q_{0}$ is an even quadratic form and $q_{1}$ an odd quadratic from. If $q$ is even, then $\mathcal{S P}_{g}[q]$ is conjugate to $\mathcal{S} \mathcal{P}_{g}\left[q_{0}\right]$ in $\mathcal{M}_{g}$, on the other hand, if $q$ is odd, then $\mathcal{S P}_{g}[q]$ is conjugate to $\mathcal{S P}_{g}\left[q_{1}\right]$ in $\mathcal{M}_{g}$. Hence, for the sake of getting some information about groups $\mathcal{S} \mathcal{P}_{g}[q]$, it suffices to consider only on $\mathcal{S} \mathcal{P}_{g}\left[q_{0}\right]$ and $\mathcal{S} \mathcal{P}_{g}\left[q_{1}\right]$. The group $\mathcal{S} \mathcal{P}_{g}\left[q_{0}\right]$ is called the even spin mapping class group, and the group $\mathcal{S P}_{g}\left[q_{1}\right]$ is called the odd spin mapping class group. The spin mapping class group is defined by Harer [8], 9]. In [11, we get a system of generators for $\mathcal{S P}_{g}\left[q_{0}\right]$. In this section, we will obtain a system of generators for $\mathcal{S P}_{g}\left[q_{1}\right]$.

Let $M$ be a simply connected smooth closed oriented 4-manifold, $(M, F)$ a knotted characteristic surface and $q_{F}$ the Rokhlin quadratic form for $(M, F)$. Rokhlin 20] showed (see also [17] and [5]),

$$
\operatorname{Arf}\left(q_{F}\right) \equiv \frac{\sigma(M)-F \cdot F}{8} \bmod 2
$$

where $\sigma(M)$ is the signature of $M$. By the above formula, we can see $q_{K_{3} \# \Sigma_{g-1}}$ is an odd quadratic form. Hence, we get a system of generators for $\mathcal{S} \mathcal{P}_{g}\left[q_{K_{3} \# \Sigma_{g-1}}\right]$ from that for $\mathcal{S P}_{g}\left[q_{1}\right]$.

We introduce some notations used for describing a system of generators for $\mathcal{S} \mathcal{P}_{g}\left[q_{1}\right]$. For a simple closed curve $a$ on $\Sigma_{g}, T_{a}$ denotes the Dehn twist about $a$. The order of composition of maps is the functional one: $T_{b} T_{a}$ means we apply $T_{a}$ first, then $T_{b}$. For elements $a, b$ and $c$ of a group, we write $\bar{c}=c^{-1}$, 
and $a * b=a b \bar{a}$. We define some elements of $\mathcal{M}_{g}$ as follows:

$$
\begin{aligned}
& C_{i}=T_{c_{i}}, B_{i}=T_{b_{i}}, B_{i}^{\prime}=T_{b_{i}^{\prime}}, \\
& X_{i}=C_{i+1} C_{i} \overline{C_{i+1}}, X_{i}^{*}=\overline{C_{i+1}} C_{i} C_{i+1} \quad(4 \leq i \leq 2 g), \\
& Y_{2 j}=C_{2 j} B_{2 j} \overline{C_{2 j}}, Y_{2 j}^{*}=\overline{C_{2 j}} B_{2 j} C_{2 j} \quad(2 \leq j \leq g-1), \\
& D_{i}=C_{i}^{2} \quad(1 \leq i \leq 2 g+1), \\
& D B_{2 j}=B_{2 j}^{2} \quad(2 \leq j \leq g-1), \\
& T_{1}=B_{4} C_{5} C_{7} \cdots C_{2 g+1} .
\end{aligned}
$$

When $g \geq 3, G_{g}$ denotes the subgroup of $\mathcal{M}_{g}$ generated by $C_{1}, C_{2}, C_{3}, X_{i}$ $(4 \leq i \leq 2 g), Y_{2 j}(2 \leq j \leq g-1), D_{i}(1 \leq i \leq 2 g+1), D B_{2 j}(2 \leq j \leq g-1)$, and $T_{1}$. It is clear that $X_{i}^{*}$ and $Y_{2 j}^{*}$ are elements of $G_{g}$. When $g=2$, the subgroup of $\mathcal{M}_{2}$ generated by $C_{1}, C_{2}, C_{3}, X_{4}$, and $D_{j}(1 \leq j \leq 5)$ is denoted by $G_{2}$. For two simple closed curves $l$ and $m$ on $\Sigma_{g}, l$ and $m$ are called $G_{g}$-equivalent (denoted by $l \underset{G_{g}}{\widetilde{m}} m$ ) if there is an element $\phi$ of $G_{g}$ such that $\phi(l)=m$.

We show that $G_{g}=\mathcal{S} \mathcal{P}_{g}\left[q_{1}\right]$. That is to say, we show,

Theorem 6.1 If $g=2, \mathcal{S P}_{2}\left[q_{1}\right]$ is generated by $C_{1}, C_{2}, C_{3}, X_{4}$, and $D_{j}$ $(1 \leq j \leq 5)$. If $g \geq 3, \mathcal{S P}_{g}\left[q_{1}\right]$ is generated by $C_{1}, C_{2}, C_{3}, X_{i}(4 \leq i \leq 2 g)$, $Y_{2 j}(2 \leq j \leq g-1), D_{k}(1 \leq k \leq 2 g+1), D B_{2 l}(2 \leq l \leq g-1)$, and $T_{1}$.

We prove Theorem 6.1 by using the same method as in the proof of Theorem 3.1 in 11. By an easy calculation, we can check that each generator of $G_{g}$ is an element of $\mathcal{S P}_{g}\left[q_{1}\right]$, therefore, $G_{g} \subset \mathcal{S} \mathcal{P}_{g}\left[q_{1}\right]$. Hence, we should show $\mathcal{S P}_{g}\left[q_{1}\right]$ $\subset G_{g}$. In the case where $g=2$, we use the Reidemeister-Schreier method to show $\mathcal{S P}_{g}\left[q_{1}\right] \subset G_{2}$ (6.4). In the case where $g \geq 3$, we use other method to show $\mathcal{S} \mathcal{P}_{g}\left[q_{1}\right] \subset G_{g}$. Here, we present this method in outline.

The integral symplectic group is denoted by $\operatorname{Sp}(2 g, \mathbb{Z})$ and the $\mathbb{Z}_{2}$ symplectic group by $\operatorname{Sp}\left(2 g, \mathbb{Z}_{2}\right)$. The generators of these groups are known (on $\operatorname{Sp}(2 g, \mathbb{Z})$ see for example [12], on $\operatorname{Sp}\left(2 g, \mathbb{Z}_{2}\right)$ see for example [7, Chap.3]), and these generators are induced by the action of $\mathcal{M}_{g}$ on $H_{1}\left(\Sigma_{g}, \mathbb{Z}\right)$ or $H_{1}\left(\Sigma_{g}, \mathbb{Z}_{2}\right)$. Therefore, the homomorphism $\Phi: \mathcal{M}_{g} \rightarrow \operatorname{Sp}(2 g, \mathbb{Z})$, defined by the action of $\mathcal{M}_{g}$ on $H_{1}\left(\Sigma_{g}, \mathbb{Z}\right)$, is a surjection, and $\Psi: \operatorname{Sp}(2 g, \mathbb{Z}) \rightarrow \operatorname{Sp}\left(2 g, \mathbb{Z}_{2}\right)$, defined by changing the coefficient from $\mathbb{Z}$ to $\mathbb{Z}_{2}$, is a surjection. In 6.1 we show $\operatorname{ker} \Phi \subset G_{g}$. In 6.2. we introduce a finite system of generators for $\operatorname{ker} \Psi$, and, for each generator, we show that one of its inverse by $\Phi$ is an element of $G_{g}$. Hence, we conclude $\operatorname{ker} \Psi \circ \Phi \subset G_{g}$. In 6.3 we introduce a finite system of generators 
for $\Psi \circ \Phi\left(\mathcal{S} \mathcal{P}_{g}\left[q_{1}\right]\right)$, and, for each generator, we show that one of its inverse by $\Psi \circ \Phi$ is an element of $G_{g}$. As a consequence, we show $\mathcal{S P}_{g}\left[q_{1}\right] \subset G_{g}$.

\subsection{Step 1 for the case where $g \geq 3$}

There is a natural surjection $\Phi: \mathcal{M}_{g} \rightarrow \operatorname{Sp}(2 g, \mathbb{Z})$ defined by the action of $\mathcal{M}_{g}$ on $H_{1}\left(\Sigma_{g} ; \mathbb{Z}\right)$. The kernel of $\Phi$ is denoted by $\mathcal{I}_{g}$ and called the Torelli group. In this subsection, we prove the following lemma:

Lemma 6.2 The Torelli group $\mathcal{I}_{g}$ is a subgroup of $G_{g}$.

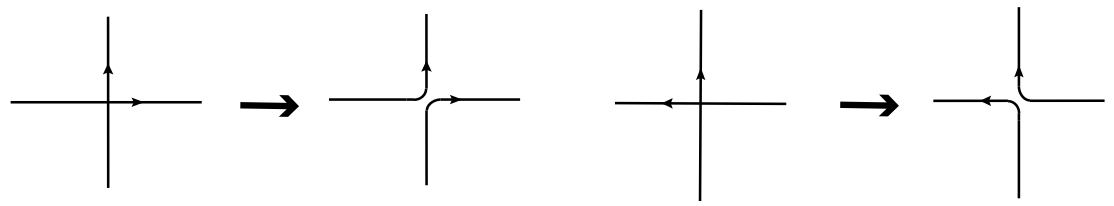

Figure 10

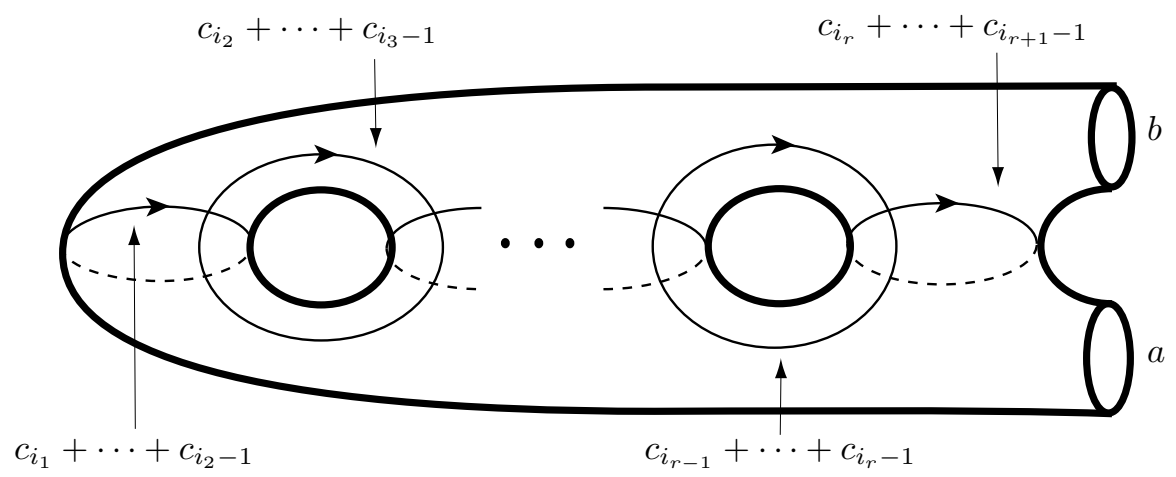

Figure 11

Johnson [14] showed that, when $g$ is larger than or equal to $3, \mathcal{I}_{g}$ is finitely generated. We review his result. For oriented simple closed curves shown in Figure [7] we refer to $\left(c_{1}, c_{2}, \ldots, c_{2 g+1}\right)$ and $\left(c_{\beta}, c_{5}, \ldots, c_{2 g+1}\right)$ as chains. For oriented simple closed curves $d$ and $e$ which intersect transversely in one point, we construct an oriented simple closed curve $d+e$ from $d \cup e$ as follows: choose a disk neighborhood of the intersection point and in it make a replacement as indicated in Figure 10. For a consecutive subset $\left\{c_{i}, c_{i+1}, \ldots, c_{j}\right\}$ of a chain, let $c_{i}+\cdots+c_{j}$ be the oriented simple closed curve constructed by repeated applications of the above operations. Let $\left(i_{1}, \ldots, i_{r+1}\right)$ be a subsequence of 
$(1,2, \ldots, 2 g+2)($ resp. $(\beta, 5, \ldots, 2 g+2))$. We construct the union of circles $\mathcal{C}$ $=\left(c_{i_{1}}+\cdots+c_{i_{2}-1}\right) \cup\left(c_{i_{2}}+\cdots+c_{i_{3}-1}\right) \cup \cdots \cup\left(c_{i_{r}}+\cdots+c_{i_{r+1}-1}\right)$. If $r$ is odd, a regular neighborhood of $\mathcal{C}$ is homeomorphic to the compact surface indicated in Figure 11 whose boundaries are $a$ and $b$. Let $\phi=T_{b} T_{a}^{-1}$, then $\phi$ is an element of $\mathcal{I}_{g}$. We denote $\phi$ by $\left[i_{1}, \ldots, i_{r+1}\right]$, and call this the odd subchain map of $\left(c_{1}, c_{2}, \ldots, c_{2 g+1}\right)$ (resp. $\left.\left(c_{\beta}, c_{5}, \ldots, c_{2 g+1}\right)\right)$ with length $r+1$. Johnson [14] showed the following theorem:

Theorem 6.3 14, Main Theorem] For $g \geq 3$, the odd subchain maps of the two chains $\left(c_{1}, c_{2}, \ldots, c_{2 g+1}\right)$ and $\left(c_{\beta}, c_{5}, \ldots, c_{2 g+1}\right)$ generate $\mathcal{I}_{g}$.

We use the following results by Johnson [14].

Lemma 6.4 14 (a) $C_{j}$ commutes with $\left[i_{1}, i_{2}, \cdots\right]$ if and only if $j$ and $j+1$ are either both contained in or are disjoint from the $i$ 's.

(b) If $i \neq j+1$, then $\overline{C_{j}} *[\cdots, j, i, \cdots]=[\cdots, j+1, i, \cdots]$.

(c) If $k \neq j$, then $C_{j} *[\cdots, k, j+1, \cdots]=[\cdots, k, j, \cdots]$.

(d) $[1,2,3,4][1,2,5,6, \ldots, 2 n] B_{4} *[3,4,5, \ldots, 2 n]=[5,6, \ldots, 2 n][1,2,3,4, \ldots$, $2 n$ ], where $3 \leq n \leq g$.

Remark 6.5 Johnson showed (d) only in the case where $n=g$. But we can apply the proof of Lemma 10 of [14] for the case where $3 \leq n<g$, since we can regard each surfaces in Figure 18 of [14] as a surface of genus $n$ which is a submanifold of $\Sigma_{g}$.

We prove that any odd subchain map of $\left(c_{1}, c_{2}, c_{3}, \ldots, c_{2 g+1}\right)$ or $\left(c_{\beta}, c_{5}, c_{6}, \ldots\right.$, $c_{2 g}$ ) is a product of elements of $G_{g}$. The following lemma shows that any odd subchain map of $\left(c_{\beta}, c_{5}, c_{6}, \ldots, c_{2 g}\right)$ is a product of an odd subchain map of $\left(c_{1}, c_{2}, c_{3}, \ldots, c_{2 g+1}\right)$ and elements of $G_{g}$.

Lemma 6.6 For any odd subchain map $h$ of $\left(c_{\beta}, c_{5}, c_{6}, \ldots, c_{2 g+1}\right)$, there is an element $g$ of $G_{g}$ such that $g * h$ is an odd subchain map of $\left(c_{1}, c_{2}, c_{3}, \ldots, c_{2 g+1}\right)$.

Proof If there is not $\beta$ in the sequence which define $h$, then $h$ is an odd subchain map of $\left(c_{1}, c_{2}, c_{3}, \ldots, c_{2 g+1}\right)$. Hence, it suffices to treat the case where the sequence defining $h$ includes $\beta$. If $g=C_{2 g+1}^{\epsilon_{2 g+1}} \cdots C_{7}^{\epsilon_{7}} C_{5}^{\epsilon_{5}} B^{-1}\left(\epsilon_{i}= \pm 1\right)$, then, under any choice of signs of $\epsilon_{i}, g \in G_{g}$. We can choose signs of $\epsilon_{i}$ so that $g * h$ is an odd subchain map of $\left(c_{1}, c_{2}, c_{3}, \ldots, c_{2 g+1}\right)$. 
From here to the end of this subsection, odd subchain maps mean only those of $\left(c_{1}, c_{2}, c_{3}, \ldots, c_{2 g+1}\right)$. The following lemma shows that any odd subchain map, whose length is at least 5 and which begins from $1,2,3,4,5$, is a product of shorter odd subchain maps and elements of $G_{g}$.

Lemma 6.7 For any $6 \leq n_{6}<n_{7}<\cdots<n_{2 k} \leq 2 g+2$,

$$
\begin{gathered}
\left(C_{4}^{2}\right) *[1,2,3,5]\left[1,2,4, n_{6}, n_{7}, \ldots, n_{2 k}\right]\left(C_{4} B_{4} \overline{C_{4}}\right) *\left[3,4,5, n_{6}, n_{7}, \ldots, n_{2 k}\right]= \\
=\left[4, n_{6}, n_{7}, \ldots, n_{2 k}\right]\left[1,2,3,4,5, n_{6}, n_{7}, \ldots, n_{2 k}\right]
\end{gathered}
$$

Proof By (a) of Lemma 6.4 $\overline{C_{4}} *[3,4,5, \ldots, 2 k]=[3,4,5, \ldots, 2 k]$, and by (d) of Lemma 6.4.

$$
\begin{aligned}
{[1,2,3,4][1,2,5,6, \ldots, 2 k] } & \cdot\left(B_{4} \overline{C_{4}}\right) *[3,4,5, \ldots, 2 k]= \\
= & {[5,6, \ldots, 2 k][1,2,3,4, \ldots, 2 k] . }
\end{aligned}
$$

By applying $C_{4}$ to the above equation and remarking that $C_{4} *[1,2,3,4]=$ $\left(C_{4}^{2}\right) *\left(\overline{C_{4}} *[1,2,3,4]\right)=\left(C_{4}^{2}\right) *[1,2,3,5]$, we get,

$$
\begin{aligned}
\left(C_{4}^{2}\right) *[1,2,3,5] \cdot[1,2,4,6, \cdots, 2 k] \cdot & \left(C_{4} B_{4} \overline{C_{4}}\right) *[3,4,5,6, \cdots, 2 k]= \\
& =[4,6,7, \cdots, 2 k][1,2,3,4,5,6, \cdots, 2 k] .
\end{aligned}
$$

After proper applications of $\overline{C_{6}}, \overline{C_{7}}, \ldots, \overline{C_{2 g+1}}$, we get the equation we need.

Lemma 6.8 (1) When $i-k \geq 3,\left(\overline{C_{i-1}} C_{i-2} C_{i-1}\right) *[\ldots, k, i, j, \ldots]=[\ldots, k, i-$ $2, j, \ldots]$.

(2) When $i-k \geq 2,\left(C_{i} C_{i-1} \overline{C_{i}}\right) *[\ldots, k, i, i+1, \ldots]=[\ldots, k, i-1, i, \ldots]$.

Proof Lemma 6.4 shows (1) and (2).

For any odd subchain map $\left[i_{1}, i_{2}, \ldots, i_{r}\right]$, we introduce a notation $\left[\left[\tau_{1}, \tau_{2}, \ldots\right.\right.$, $\left.\left.\tau_{2 g+2}\right]\right]: \tau_{k}=1$ if $k$ is a member of $\left\{i_{1}, i_{2}, \ldots, i_{r}\right\}$, and $\tau_{k}=0$ if $k$ is not a member of $\left\{i_{1}, i_{2}, \ldots, i_{r}\right\}$. For $\left[\left[\tau_{1}, \tau_{2}, \ldots, \tau_{2 g+2}\right]\right], \tau_{i}(1 \leq i \leq 2 g+2)$ is called the $i$-th tack of $\left[\left[\tau_{1}, \tau_{2}, \ldots, \tau_{2 g+2}\right]\right]$, and if $\tau_{i}=0$ (resp. 1$)$ then $\tau_{i}$ is called a 0 -tack (resp. a 1 -tack). The number of 1 -tacks in $\left[\left[\tau_{1}, \tau_{2}, \ldots, \tau_{2 g+2}\right]\right]$ is called the length of $\left[\left[\tau_{1}, \tau_{2}, \ldots, \tau_{2 g+2}\right]\right]$. Lemma 6.8 (1) means that, when $k \geq 3$, if there is a sequence of 0 -tacks which begins from the $k+1$-st tack and whose length is at least 2 , then the 1 -tack subsequent to this 0 -tack sequence is moved to left by 2-steps under the action of $G_{g}$. Lemma 6.8 (2) means that, when $k \geq 3$, if there is a sequence of 0 -tacks which begins from the $k+1$-st tack and 
whose length is at least 1 , then the adjacent two 1 -tacks subsequent to this 0 -tack sequence is moved to left by 1 -step under the action of $G_{g}$. Therefore, for any $\left[\left[\tau_{1}, \tau_{2}, \ldots, \tau_{2 g+2}\right]\right]$, we see,

$$
\left[\left[\tau_{1}, \tau_{2}, \ldots, \tau_{2 g+2}\right]\right] \underset{G_{g}}{\sim}\left[\left[\tau_{1}, \tau_{2}, \tau_{3}, 1, \ldots, 1,0,1, \ldots, 0,1,0, \ldots, 0\right]\right],
$$

where $1, \ldots, 1$ is a sequence of 1 -tacks ( $b$ denotes the length of this sequence), $0,1, \ldots, 0,1$ is a sequence arranged 0 -tacks and 1 -tacks alternatively ( $t$ denotes the number of 1 -tacks in this sequence ), $0, \ldots, 0$ is a sequence of 0 -tacks. Since $C_{1}, C_{2}, C_{3} \in G_{g}$, if there is one 1-tack among $\tau_{1}, \tau_{2}, \tau_{3}$, then $\left[\left[\tau_{1}, \tau_{2}, \tau_{3}, \ldots\right]\right] \widetilde{G}_{g}$ $[[1,0,0, \ldots]]$, if there are two 1 -tacks among $\tau_{1}, \tau_{2}, \tau_{3}$, then $\left[\left[\tau_{1}, \tau_{2}, \tau_{3}, \ldots\right]\right]{\widetilde{G_{g}}}^{\sigma_{g}}$ $[[1,1,0, \ldots]]$. The number of 1 -tacks in $\tau_{1}, \tau_{2}, \tau_{3}$ is denoted by $h$.

Lemma 6.9 Any odd subchain map is a product of elements of $G_{g}$ and the odd subchain maps whose $h$ and $b$ are (1) $h=3, b=1$, (2) $h=3, b=0$, (3) $h=2, b=0$, (4) $h=1, b=0$, (5) $h=0, b=0$.

Proof We treat the case where $h=3$. If $b \geq 2$, by Lemma 6.7 this odd subchain map is a product of elements of $G_{g}$ and shorter odd subchain maps.

We treat the case where $h=2$. If $b \geq 3$,

$$
[[1,1,0,1,1,1, \ldots]] \underset{C_{3}}{\longrightarrow}[[1,1,1,0,1,1, \ldots]] \underset{\text { Lemma } \underset{\underline{6.8}(2)}{\longrightarrow}}{\longrightarrow}[[1,1,1,1,1,0, \ldots]],
$$

by Lemma 6.7 the last odd subchain map is a product of elements of $G_{g}$ and shorter odd subchain maps. If $b=2$,

$$
[[1,1,0,1,1,0, \ldots]] \underset{C_{3}}{\longrightarrow}[[1,1,1,0,1,0, \ldots]],
$$

the last odd subchain map is in the case where $h=3, b=0$. If $b=1, t$ should be at least 1 , and

$$
\begin{aligned}
{[[1,1,0,1,0,1,0, \ldots]] \underset{C_{3}}{\longrightarrow} } & {[[1,1,1,0,0,1,0, \ldots]] } \\
& \underset{\text { Lemma }[\underline{6.8} 1)}{\longrightarrow}[[1,1,1,1,0,0,0, \ldots]],
\end{aligned}
$$

the last odd subchain map is in the case where $h=3, b=1$.

We treat the case where $h=1$. If $b \geq 5$,

$$
\begin{aligned}
& {[[1,0,0,1,1,1,1,1, \ldots]] \underset{C_{2} C_{3}}{\longrightarrow}[[1,1,0,0,1,1,1,1, \ldots]]} \\
& \underset{\text { Lemma } \underset{6.8]}{\longrightarrow} 2)}{\longrightarrow}[[1,1,0,1,1,0,1,1, \ldots]] \underset{\text { Lemma }[6.8(2)}{\longrightarrow}[[1,1,0,1,1,1,1,0, \ldots]] \\
& \underset{C_{3}}{\longrightarrow}[[1,1,1,0,1,1,1,0, \ldots]] \underset{\text { Lemma }[\underline{6.8}(2)}{\longrightarrow}[[1,1,1,1,1,0,1,0, \ldots]],
\end{aligned}
$$


by Lemma 6.7 the last odd subchain map is a product of elements of $G_{g}$ and the shorter odd subchain maps. If $b=4$,

$$
\begin{aligned}
& {[[1,0,0,1,1,1,1,0, \ldots]] \underset{C_{2} C_{3}}{\longrightarrow}[[1,1,0,0,1,1,1,0, \ldots]]} \\
& \underset{\text { Lemma } \underset{6.8}{\longrightarrow} 2)}{\longrightarrow}[[1,1,0,1,1,0,1,0, \ldots]] \underset{C_{3}}{\longrightarrow}[[1,1,1,0,1,0,1,0, \ldots]],
\end{aligned}
$$

the last odd subchain map is in the case where $h=3, b=0$. If $b=3$ and $t=0$,

$$
\begin{aligned}
& {[[1,0,0,1,1,1,0,0, \ldots]] \underset{C_{2} C_{3}}{\longrightarrow}[[1,1,0,0,1,1,0,0, \ldots]]} \\
& \underset{\text { Lemma } \underset{6.8}{\longrightarrow} 2)}{\longrightarrow}[[1,1,0,1,1,0,0,0, \ldots]] \underset{C_{3}}{\longrightarrow}[[1,1,1,0,1,0,0,0, \ldots]],
\end{aligned}
$$

the last odd subchain map is in the case where $h=3, b=0$. If $b=3$ and $t \geq 2$,

$$
\begin{aligned}
& {[[1,0,0,1,1,1,0,1, \ldots]] \underset{C_{2} C_{3}}{\longrightarrow}[[1,1,0,0,1,1,0,1, \ldots]]} \\
& \underset{\text { Lemma } \underset{6.8}{\longrightarrow} 2)}{\longrightarrow}[[1,1,0,1,1,0,0,1, \ldots]] \underset{\text { Lemma } 2[6.8(1)}{\longrightarrow}[[1,1,0,1,1,1,0,0, \ldots]] \\
& \underset{C_{3}}{\longrightarrow}[[1,1,1,0,1,1,0,0, \ldots]] \underset{\text { Lemma } \underset{6.8}{\longrightarrow}(2)}{\longrightarrow}[[1,1,1,1,1,0,0,0, \ldots]],
\end{aligned}
$$

by Lemma 6.7 the last odd subchain map is a product of elements of $G_{g}$ and shorter odd subchain maps. If $b=2$,

$$
[[1,0,0,1,1,0, \ldots]] \underset{C_{2} C_{3}}{\longrightarrow}[[1,1,0,0,1,0, \ldots]],
$$

the last odd subchain map is in the case where $h=2, b=0$. If $b=1, t$ should be at least 2 ,

$$
\begin{aligned}
& {[[1,0,0,1,0,1,0,1, \ldots]] \underset{C_{2} C_{3}}{\longrightarrow}[[1,1,0,0,0,1,0,1, \ldots]]} \\
& \underset{\text { Lemma } \underset{6.8(1)}{\longrightarrow}}{\longrightarrow}[[1,1,0,1,0,1,0,0, \ldots]],
\end{aligned}
$$

the last odd subchain map is in the case where $h=2, b=1$, which we treat before.

We treat the case where $h=0$. If $b \geq 7$,

$$
\begin{aligned}
& {[[0,0,0,1,1,1,1,1,1,1, \ldots]] \underset{C_{1} C_{2} C_{3}}{\longrightarrow}[[1,0,0,0,1,1,1,1,1,1, \ldots]]} \\
& \underset{\text { Lemma } \underset{6.8}{\longrightarrow}(2)}{\longrightarrow}[[1,0,0,1,1,1,1,1,1,0, \ldots]] \underset{C_{2} C_{3}}{\longrightarrow}[[1,1,0,0,1,1,1,1,1,0, \ldots]] \\
& \underset{\text { Lemma } \underset{6.8}{\longrightarrow} 2)}{\longrightarrow}[[1,1,0,1,1,1,1,0,1,0, \ldots]] \underset{C_{3}}{\longrightarrow}[[1,1,1,0,1,1,1,0,1,0, \ldots]] \\
& \underset{\text { Lemma } \underset{6.8}{\longrightarrow}(2)}{\longrightarrow}[[1,1,1,1,1,0,1,0,1,0, \ldots]] \text {, }
\end{aligned}
$$


by Lemma 6.7 the last odd subchain map is a product of $G_{g}$ and shorter odd subchain maps. If $b=6$,

$$
\begin{aligned}
& {[[0,0,0,1,1,1,1,1,1,0, \ldots]] \underset{C_{1} C_{2} C_{3}}{\longrightarrow}[[1,0,0,0,1,1,1,1,1,0, \ldots]]} \\
& \underset{\text { Lemma } \underset{6.8}{\longrightarrow}(2)}{\longrightarrow}[[1,0,0,1,1,1,1,0,1,0, \ldots]] \underset{C_{2} C_{3}}{\longrightarrow}[[1,1,0,0,1,1,1,0,1,0, \ldots]] \\
& \underset{\text { Lemma } \underset{6.8}{\longrightarrow}(2)}{\longrightarrow}[[1,1,0,1,1,0,1,0,1,0, \ldots]] \underset{C_{3}}{\longrightarrow}[[1,1,1,0,1,0,1,0,1,0, \ldots]],
\end{aligned}
$$

the last odd subchain map is in the case where $h=3, b=0$. If $b=5, t$ should be at least 1 and,

$$
\begin{aligned}
& {[[0,0,0,1,1,1,1,1,0,1, \ldots]] \underset{C_{1} \vec{C}_{2} C_{3}}{\longrightarrow}[[1,0,0,0,1,1,1,1,0,1, \ldots]]} \\
& \underset{\operatorname{Lemma}[6.8(2)}{\longrightarrow}[[1,0,0,1,1,1,1,0,0,1, \ldots]] \underset{\text { Lemma }[6.8(1)}{\longrightarrow}[[1,0,0,1,1,1,1,1,0,0, \ldots]] \\
& \underset{C_{2} C_{3}}{\longrightarrow}[[1,1,0,0,1,1,1,1,0,0, \ldots]] \underset{\text { Lemma } 6.8(2)}{\longrightarrow}[[1,1,0,1,1,1,1,0,0,0, \ldots]] \\
& \underset{C_{3}}{\longrightarrow}[[1,1,1,0,1,1,1,0,0,0, \ldots]] \underset{\text { Lemma }[6.8(2)}{\longrightarrow}[[1,1,1,1,1,0,1,0,0,0, \ldots]],
\end{aligned}
$$

by Lemma 6.7 the last odd subchain map is a product of elements of $G_{g}$ and shorter odd subchain maps. If $b=4$,

$$
\begin{aligned}
& {[[0,0,0,1,1,1,1,0, \ldots]] \underset{C_{1} C_{2} C_{3}}{\longrightarrow}[[1,0,0,0,1,1,1,0, \ldots]]} \\
& \underset{\text { Lemma } \underset{\underline{6.8}(2)}{\longrightarrow}}{\longrightarrow}[[1,0,0,1,1,0,1,0, \ldots]] \underset{C_{2} C_{3}}{\longrightarrow}[[1,1,0,0,1,0,1,0, \ldots]],
\end{aligned}
$$

the last odd subchain map is in the case where $h=2, b=0$. If $b=3$ and $t=1$,

$$
\begin{aligned}
& {[[0,0,0,1,1,1,0,1,0, \ldots]] \underset{C_{1}}{\overrightarrow{C_{2} C_{3}}}[[1,0,0,0,1,1,0,1,0, \ldots]]} \\
& \underset{\operatorname{Lemma[6.8}(2)}{\longrightarrow}[[1,0,0,1,1,0,0,1,0, \ldots]] \underset{\text { Lemma } \underset{6.8}{\longrightarrow}(1)}{\longrightarrow}[[1,0,0,1,1,1,0,0,0, \ldots]] \\
& \underset{C_{2} C_{3}}{\longrightarrow}[[1,1,0,0,1,1,0,0,0, \ldots]] \underset{\text { Lemma } \underset{6.8]}{\longrightarrow} 2)}{\longrightarrow}[[1,1,0,1,1,0,0,0,0, \ldots]] \\
& \underset{C_{3}}{\longrightarrow}[[1,1,1,0,1,0,0,0,0, \ldots]],
\end{aligned}
$$

the last odd subchain map is in the case where $h=3, b=0$. If $b=3$ and $t \neq 1$, then $t$ should be at least 3 and,

$$
\begin{aligned}
& {[[0,0,0,1,1,1,0,1,0,1,0,1, \ldots]]} \\
& \underset{C_{1} C_{2} C_{3}}{\longrightarrow}[[1,0,0,0,1,1,0,1,0,1,0,1, \ldots]] \\
& \underset{\text { Lemma } \underset{6.8}{6} 2)}{\longrightarrow}[[1,0,0,1,1,0,0,1,0,1,0,1, \ldots]]
\end{aligned}
$$




$$
\begin{aligned}
& \underset{\text { Lemma } \underset{6.8}{*} 1)}{\longrightarrow}[[1,0,0,1,1,1,0,1,0,1,0,0, \ldots]] \\
& \underset{C_{2} C_{3}}{\longrightarrow}[[1,1,0,0,1,1,0,1,0,1,0,0, \ldots]] \\
& \underset{\text { Lemma } \underset{6.8]}{\longrightarrow} 2)}{\longrightarrow}[[1,1,0,1,1,0,0,1,0,1,0,0, \ldots]]
\end{aligned}
$$

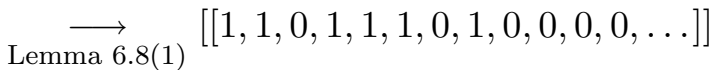

$$
\begin{aligned}
& \underset{C_{3}}{\longrightarrow}[[1,1,1,0,1,1,0,1,0,0,0,0, \ldots]] \\
& \underset{\text { Lemma } \underset{[6.8]}{\longrightarrow} 2)}{\longrightarrow}[[1,1,1,1,1,0,0,1,0,0,0,0, \ldots]],
\end{aligned}
$$

by Lemma 6.7 the last odd subchain map is a product of elements of $G_{g}$ and shorter odd subchain maps. If $b=2$,

$$
[[0,0,0,1,1,0, \ldots]] \underset{C_{1} \underset{C_{2} C_{3}}{\longrightarrow}}{\longrightarrow}[[1,0,0,0,1,0, \ldots]],
$$

the last odd subchain map is in the case where $h=1, b=0$. If $b=1$, then $t$ should be at least 3 and,

$$
\begin{aligned}
& {[[0,0,0,1,0,1,0,1,0,1, \ldots]] \underset{C_{1} C_{2} C_{3}}{\longrightarrow}[[1,0,0,0,0,1,0,1,0,1, \ldots]]} \\
& \underset{\text { Lemma } \underset{6.8}{6}(1)}{\longrightarrow}[[1,0,0,1,0,1,0,1,0,0, \ldots]] \underset{C_{2} C_{3}}{\longrightarrow}[[1,1,0,0,0,1,0,1,0,0, \ldots]] \\
& \underset{\text { Lemma } \underset{6.8}{\longrightarrow} 1)}{\longrightarrow}[[1,1,0,1,0,1,0,0,0,0, \ldots]] \underset{C_{3}}{\longrightarrow}[[1,1,1,0,0,1,0,0,0,0, \ldots]] \\
& \underset{\text { Lemma } \underset{6.8}{\longrightarrow}(1)}{\longrightarrow}[[1,1,1,1,0,0,0,0,0,0, \ldots]],
\end{aligned}
$$

the last odd subchain map is in the case where $h=3, b=1$.

This Lemma follows from the above case by case arguments and the induction on the length $(=h+b+t)$ of odd subchain maps.

Lemma 6.10 Any odd subchain maps of the 6 cases listed in Lemma 6.9 are products of elements of $G_{g}$ and odd subchain maps $[[1,1,1,1,0, \ldots, 0]]$, $[[1,1,1,0,1,0, \ldots, 0]],[[1,1,1,0,1,0,1,0,1,0, \ldots, 0]]$, and $[[1,1,0,0,1,0,1,0$, $\ldots, 0]]$, where $0, \ldots, 0$ are sequences of 0 -tacks.

Proof By checking figures of chain maps, for examples $[[1,1,1,1,0,1,0,1, \ldots]]$ indicated in Figure 12 and $[[0,0,0,0,1,0,1,0, \ldots]]$ indicated in Figure 13] we see that if a odd subchain map begins from $[[0,0,0,0, \ldots$, or $[[1,1,1,1, \ldots$, then this map commutes with $B_{4}$, hence $B_{4} *$ does not effect on this map. 


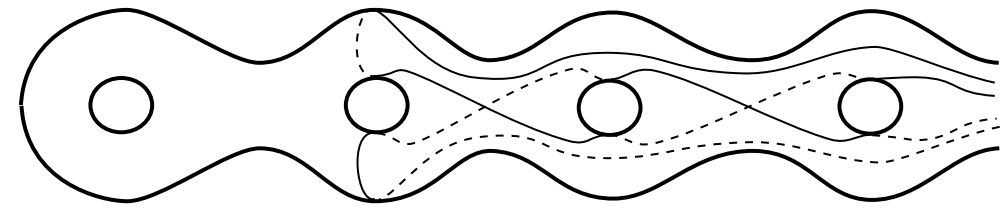

Figure 12

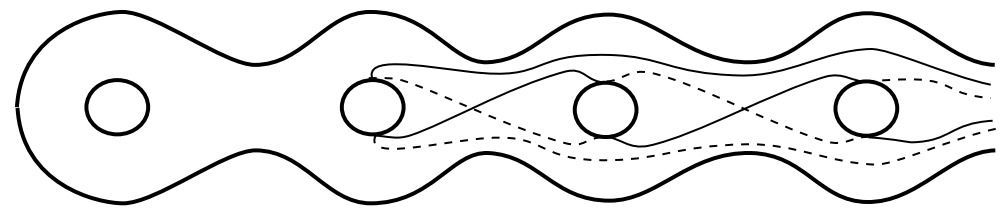

Figure 13

We treat the case where $h=3, b=1$. If $t=0$, then this odd subchain map is $[[1,1,1,1,0, \ldots, 0]]$. If $t \neq 0$, then $t$ should be at least 2 and,

$$
[[1,1,1,1,0,1,0,1, \ldots]] \underset{T_{1}}{\longrightarrow}[[1,1,1,1,1,0,1,0, \ldots]]
$$

by Lemma 6.7 the last odd subchain map is a product of elements of $G_{g}$ and shorter odd subchain maps.

We treat the case where $h=3, b=0$. In this case, $t$ should be an odd integer at least 1 . If $t=1$, then this map is $[[1,1,1,0,1,0, \ldots, 0]]$. If $t=3$, then this map is $[[1,1,1,0,1,0,1,0,1,0, \ldots, 0]]$. If $t \geq 5$,

$$
\begin{aligned}
& {[[1,1,1,0,1,0,1,0,1,0,1,0,1,0, \ldots]]} \\
& \underset{\overline{C_{3}}}{\longrightarrow}[[1,1,0,1,1,0,1,0,1,0,1,0,1,0, \ldots]] \\
& \underset{\text { Lemma } \underset{6.8}{6}(2)}{\longrightarrow}[[1,1,0,0,1,1,1,0,1,0,1,0,1,0, \ldots]]
\end{aligned}
$$

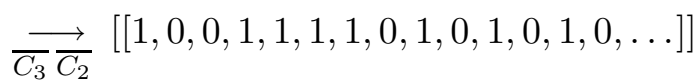

$$
\begin{aligned}
& \underset{\text { Lemma } \underset{6.8}{\longrightarrow}(2)}{\longrightarrow}[[1,0,0,0,1,1,1,1,1,0,1,0,1,0, \ldots]] \\
& \overline{C_{3}} \underset{C_{2}}{\longrightarrow}[[0,0,0,1,1,1,1,1,1,0,1,0,1,0, \ldots]] \\
& \underset{\text { Lemma } \underset{6.8]}{2}(2)}{\longrightarrow}[[0,0,0,0,1,1,1,1,1,1,1,0,1,0, \ldots]] \\
& \underset{\overline{T_{1}}}{\longrightarrow}[[0,0,0,0,1,1,1,1,1,1,0,1,0,1, \ldots]] \\
& \underset{\text { Lemma } \underset{6.8]}{6}(2)}{\longrightarrow}[[0,0,0,1,1,1,1,1,1,0,0,1,0,1, \ldots]]
\end{aligned}
$$




$$
\begin{aligned}
& \underset{C_{1} C_{2} C_{3}}{\longrightarrow}[[1,0,0,0,1,1,1,1,1,0,0,1,0,1, \ldots]] \\
& \underset{\text { Lemma } \underset{6.8}{\longrightarrow}(1)}{\longrightarrow}[[1,0,0,0,1,1,1,1,1,1,0,0,0,1, \ldots]] \\
& \underset{\text { Lemma } \underset{6.8]}{2}(2)}{\longrightarrow}[[1,0,0,1,1,1,1,1,1,0,0,0,0,1, \ldots]] \\
& \underset{C_{2} C_{3}}{\longrightarrow}[[1,1,0,0,1,1,1,1,1,0,0,0,0,1, \ldots]] \\
& \underset{\text { Lemma } \underset{6.8]}{2}(2)}{\longrightarrow}[[1,1,0,1,1,1,1,0,1,0,0,0,0,1, \ldots]] \\
& \underset{C_{3}}{\longrightarrow}[[1,1,1,0,1,1,1,0,1,0,0,0,0,1, \ldots]] \\
& \underset{\text { Lemma } \underset{[6.8}{\longrightarrow} 2)}{\longrightarrow}[[1,1,1,1,1,0,1,0,1,0,0,0,0,1, \ldots]],
\end{aligned}
$$

by Lemma 6.7. the last odd subchain map is a product of elements of $G_{g}$ and shorter odd subchain maps.

We treat the case where $h=2, b=0$. In this case, $t$ should be even integer at least 2 . If $t=2$, this map is $[[1,1,0,0,1,0,1,0, \ldots, 0]]$. If $t \geq 4$,

$$
\begin{aligned}
& {[[1,1,0,0,1,0,1,0,1,0,1,0, \ldots]]} \\
& \underset{C_{3}}{\longrightarrow} \underset{C_{2}}{\longrightarrow}[[1,0,0,1,1,0,1,0,1,0,1,0, \ldots]] \\
& \underset{\text { Lemma } \underset{6.8}{2} 2)}{\longrightarrow}[[1,0,0,0,1,1,1,0,1,0,1,0, \ldots]] \\
& \overline{C_{3}} \underset{\overline{C_{2}}}{\overrightarrow{C_{1}}}[[0,0,0,1,1,1,1,0,1,0,1,0, \ldots]] \\
& \underset{\text { Lemma } \underset{6.8]}{2} 2)}{\longrightarrow}[[0,0,0,0,1,1,1,1,1,0,1,0, \ldots]] \\
& \underset{\overline{T_{1}}}{\longrightarrow}[[0,0,0,0,1,1,1,1,0,1,0,1, \ldots]] \\
& \underset{\text { Lemma } \underset{6.8]}{2} 2)}{\longrightarrow}[[0,0,0,1,1,1,1,0,0,1,0,1, \ldots]] \\
& \underset{C_{1} C_{2} C_{3}}{\longrightarrow}[[1,0,0,0,1,1,1,0,0,1,0,1, \ldots]] \\
& \underset{\text { Lemma[6.8] } 1)}{\longrightarrow}[[1,0,0,0,1,1,1,1,0,1,0,0, \ldots]] \\
& \underset{\text { Lemma } \underset{6.8}{2} 2)}{\longrightarrow}[[1,0,0,1,1,1,1,0,0,1,0,0, \ldots]] \\
& \underset{\operatorname{Lemma}[6.81}{\longrightarrow}[1] \\
& \underset{C_{2} C_{3}}{\longrightarrow}[[1,1,0,0,1,1,1,1,0,0,0,0, \ldots]] \\
& \underset{\text { Lemma } \underset{6.8]}{\longrightarrow} 2)}{\longrightarrow}[[1,1,0,1,1,1,1,0,0,0,0,0, \ldots]]
\end{aligned}
$$




$$
\begin{aligned}
& \underset{C_{3}}{\longrightarrow}[[1,1,1,0,1,1,1,0,0,0,0,0, \ldots]] \\
& \underset{\text { Lemma } \underset{6.8}{2} 2)}{\longrightarrow}[[1,1,1,1,1,0,1,0,0,0,0,0, \ldots]],
\end{aligned}
$$

by Lemma 6.7 the last odd subchain map is a product of elements of $G_{g}$ and shorter odd subchain maps.

We treat the case where $h=1, b=0$. In this case, $t$ should be an odd integer at least 3 . If $t=3$,

$$
\begin{aligned}
& {[[1,0,0,0,1,0,1,0,1,0, \ldots]] \underset{\overline{C_{3}}}{\overrightarrow{C_{2}}} \overrightarrow{C_{1}}[[0,0,0,1,1,0,1,0,1,0, \ldots]]} \\
& \underset{\operatorname{Lemma[6.8(2)}}{\longrightarrow}[[0,0,0,0,1,1,1,0,1,0, \ldots]] \underset{\overline{T_{1}}}{\longrightarrow}[[0,0,0,0,1,1,0,1,0,1, \ldots]] \\
& \underset{\text { Lemma } \underset{6.8}{\longrightarrow}(2)}{\longrightarrow}[[0,0,0,1,1,0,0,1,0,1, \ldots]] \underset{\text { Lemma } \underset{6.8}{\longrightarrow}(1)}{\longrightarrow}[[0,0,0,1,1,1,0,1,0,0, \ldots]] \\
& \underset{C_{1} C_{2} C_{3}}{\longrightarrow}[[1,0,0,0,1,1,0,1,0,0, \ldots]] \underset{\text { Lemma }[6.8] 2)}{\longrightarrow}[[1,0,0,1,1,0,0,1,0,0, \ldots]] \\
& \underset{\text { Lemma } \underset{6.8}{\longrightarrow}(1)}{\longrightarrow}[[1,0,0,1,1,1,0,0,0,0, \ldots]] \underset{C_{2} C_{3}}{\longrightarrow}[[1,1,0,0,1,1,0,0,0,0, \ldots]] \\
& \underset{\text { Lemma } \underset{6.8}{\longrightarrow}(2)}{\longrightarrow}[[1,1,0,1,1,0,0,0,0,0, \ldots]] \underset{C_{3}}{\longrightarrow}[[1,1,1,0,1,0,0,0,0,0, \ldots]] .
\end{aligned}
$$

$$
\begin{aligned}
& {[[1,0,0,0,1,0,1,0,1,0,1,0, \ldots]]} \\
& \overline{C_{3}} \underset{\overline{C_{2}}}{\longrightarrow}[[0,0,0,1,1,0,1,0,1,0,1,0, \ldots]] \\
& \underset{\text { Lemma } \underset{6.8]}{2} 2)}{\longrightarrow}[[0,0,0,0,1,1,1,0,1,0,1,0, \ldots]] \\
& \underset{\overline{T_{1}}}{\longrightarrow}[[0,0,0,0,1,1,0,1,0,1,0,1, \ldots]] \\
& \underset{\text { Lemma } \underset{6.8]}{6} 2)}{\longrightarrow}[[0,0,0,1,1,0,0,1,0,1,0,1, \ldots]] \\
& \underset{\text { Lemma } \underset{6.8]}{1} 1)}{\longrightarrow}[[0,0,0,1,1,1,0,1,0,1,0,0, \ldots]] \\
& \underset{C_{1} C_{2} C_{3}}{\longrightarrow}[[1,0,0,0,1,1,0,1,0,1,0,0, \ldots]] \\
& \underset{\text { Lemma } \underset{6.8}{6} 2)}{\longrightarrow}[[1,0,0,1,1,0,0,1,0,1,0,0, \ldots]] \\
& \underset{\operatorname{Lemma}[6.81}{\longrightarrow}[1] \\
& \underset{C_{2} C_{3}}{\longrightarrow}[[1,1,0,0,1,1,0,1,0,0,0,0, \ldots]] \\
& \underset{\text { Lemma } \underset{6.8]}{\longrightarrow} 2)}{\longrightarrow}[[1,1,0,1,1,0,0,1,0,0,0,0, \ldots]]
\end{aligned}
$$




$$
\begin{aligned}
& \underset{\text { Lemma } \underset{6.8}{6} 1)}{\longrightarrow}[[1,1,0,1,1,1,0,0,0,0,0,0, \ldots]] \\
& \underset{C_{3}}{\longrightarrow}[[1,1,1,0,1,1,0,0,0,0,0,0, \ldots]] \\
& \underset{\text { Lemma } \underset{6.8}{\longrightarrow} 2)}{\longrightarrow}[[1,1,1,1,1,0,0,0,0,0,0,0, \ldots]],
\end{aligned}
$$

by Lemma 6.7 the last odd subchain map is a product of elements of $G_{g}$ and shorter odd subchain maps.

We treat the case where $h=0, b=0$. In this case, $t$ should be an even integer at least 4 . If $t=4$,

$$
\begin{aligned}
& {[[0,0,0,0,1,0,1,0,1,0,1,0, \ldots]]} \\
& \underset{\overline{T_{1}}}{\longrightarrow}[[0,0,0,0,0,1,0,1,0,1,0,1, \ldots]] \\
& \underset{\text { Lemma } \underset{6.8}{1} 1)}{\longrightarrow}[[0,0,0,1,0,1,0,1,0,1,0,0, \ldots]] \\
& \underset{C_{1} C_{2} C_{3}}{\longrightarrow}[[1,0,0,0,0,1,0,1,0,1,0,0, \ldots]] \\
& \underset{\text { Lemma } \underset{6.8}{\longrightarrow} 1)}{\longrightarrow}[[1,0,0,1,0,1,0,1,0,0,0,0, \ldots]] \\
& \underset{C_{2} C_{3}}{\longrightarrow}[[1,1,0,0,0,1,0,1,0,0,0,0, \ldots]] \\
& \underset{\text { Lemma } \underset{6.8}{1} 1)}{\longrightarrow}[[1,1,0,1,0,1,0,0,0,0,0,0, \ldots]] \\
& \underset{C_{3}}{\longrightarrow}[[1,1,1,0,0,1,0,0,0,0,0,0, \ldots]] \\
& \underset{\text { Lemma } \underset{6.8}{6} 1)}{\longrightarrow}[[1,1,1,1,0,0,0,0,0,0,0,0, \ldots]] .
\end{aligned}
$$

If $t \geq 6$,

$$
\begin{aligned}
& {[[0,0,0,0,1,0,1,0,1,0,1,0,1,0,1,0, \ldots]]} \\
& \underset{\overline{T_{1}}}{\longrightarrow}[[0,0,0,0,0,1,0,1,0,1,0,1,0,1,0,1, \ldots]] \\
& \underset{\text { Lemma } \underset{[6.8}{\longrightarrow}(1)}{\longrightarrow}[[1,1,1,1,0,1,0,1, \ldots]],
\end{aligned}
$$

the last odd subchain map is in the case where $h=3, b=1$, which we treat before.

Lemma 6.11 The odd subchain maps $[[1,1,1,1,0, \ldots, 0]]$, $[[1,1,1,0,1,0, \ldots$, $0]]$ and $[[1,1,0,0,1,0,1,0, \ldots, 0]]$ are elements of $G_{g}$. 
Proof In a proof of this Lemma, we use "braid relation", which is explained as follows. Let $a$ and $b$ are simple closed curves on $\Sigma_{g}$ intersecting transversely in one point, then $T_{a} T_{b} T_{a}^{-1}=T_{b}^{-1} T_{a} T_{b}$, in other word, $T_{a} * T_{b}=\overline{T_{b}} * T_{a}$.

Let $b_{4}^{\prime}$ be the simple closed curve on $\Sigma_{g}$ indicated in Figure 8 and let $B_{4}^{\prime}=T_{b_{4}^{\prime}}$. The odd subchain map $[[1,1,1,1,0, \ldots, 0]]$ is equal to $B_{4} \overline{B_{4}^{\prime}}$. Since $b_{4}^{\prime}=$ $C_{4} C_{3} C_{2} C_{1} C_{1} C_{2} C_{3} C_{4}\left(b_{4}\right)$,

$$
\begin{aligned}
B_{4} \overline{B_{4}^{\prime}}= & B_{4} C_{4} C_{3} C_{2} C_{1} C_{1} C_{2} C_{3} C_{4} \overline{B_{4}} \overline{C_{4}} \overline{C_{3}} \overline{C_{2}} \overline{C_{1}} \overline{C_{1}} \overline{C_{2}} \overline{C_{3}} \overline{C_{4}} \\
= & \left(B_{4} C_{4} C_{3} C_{2}\right) *\left(C_{1} C_{1}\right) \cdot\left(B_{4} C_{4} C_{3}\right) *\left(C_{2} C_{2}\right) \cdot\left(B_{4} C_{4}\right) *\left(C_{3} C_{3}\right) . \\
& \cdot B_{4} *\left(C_{4} C_{4}\right) \cdot\left(\overline{C_{4}} \overline{C_{3}} \overline{C_{2}}\right) *\left(\overline{C_{1}} \overline{C_{1}}\right) \cdot\left(\overline{C_{4}} \overline{C_{3}}\right) *\left(\overline{C_{2}} \overline{C_{2}}\right) . \\
& \cdot \overline{C_{4}} *\left(\overline{C_{3}} \overline{C_{3}}\right) \cdot \overline{C_{4}} \overline{C_{4}} .
\end{aligned}
$$

This equation means that $B_{4} \overline{B_{4}^{\prime}}$ is a product of squares Dehn twists. By using braid relations of $\mathcal{M}_{g}$, we can see that these squares of Dehn twists are elements of $G_{g}$ as follows,

$$
\begin{aligned}
\left(B_{4} C_{4} C_{3} C_{2}\right) *\left(C_{1} C_{1}\right) & =\left(\overline{C_{1}} \cdot \overline{C_{2}} \cdot \overline{C_{3}} \cdot B_{4}\right) *\left(C_{4} C_{4}\right) \\
& =\left(\overline{C_{1}} \cdot \overline{C_{2}} \cdot \overline{C_{3}}\right) *\left(B_{4} C_{4} \overline{B_{4}} \cdot B_{4} C_{4} \overline{B_{4}}\right), \\
\left(B_{4} C_{4} C_{3}\right) *\left(C_{2} C_{2}\right) & =\left(\overline{C_{2}} \cdot \overline{C_{3}} \cdot B_{4}\right) *\left(C_{4} C_{4}\right) \\
& =\left(\overline{C_{2}} \cdot \overline{C_{3}}\right) *\left(B_{4} C_{4} \overline{B_{4}} \cdot B_{4} C_{4} \overline{B_{4}}\right), \\
\left(B_{4} C_{4}\right) *\left(C_{3} C_{3}\right) & =\left(\overline{C_{3}} \cdot B_{4}\right) *\left(C_{4} C_{4}\right)=\overline{C_{3}} *\left(B_{4} C_{4} \overline{B_{4}} \cdot B_{4} C_{4} \overline{B_{4}}\right), \\
B_{4} *\left(C_{4} C_{4}\right) & =B_{4} C_{4} \overline{B_{4}} \cdot B_{4} C_{4} \overline{B_{4}} \\
\left(\overline{C_{4}} \overline{C_{3}} \overline{C_{2}}\right) *\left(C_{1} C_{1}\right) & =\left(C_{1} \cdot C_{2} \cdot C_{3}\right) *\left(C_{4} C_{4}\right), \\
\left(\overline{C_{4}} \overline{C_{3}}\right) *\left(C_{2} C_{2}\right) & =\left(C_{2} \cdot C_{3}\right) *\left(C_{4} C_{4}\right), \\
\overline{C_{4}} *\left(C_{3} C_{3}\right) & =C_{3} *\left(C_{4} C_{4}\right) .
\end{aligned}
$$

Since $\overline{C_{4}} *[[1,1,1,1,0, \ldots, 0]]=[[1,1,1,0,1,0, \ldots, 0]]$,

$$
\begin{aligned}
& {[[1,1,1,0,1,0, \ldots, 0]]=\overline{C_{4}} *\left(B_{4} \overline{B_{4}^{\prime}}\right)} \\
& =\left(\overline{C_{4}} B_{4} C_{4} C_{3} C_{2}\right) *\left(C_{1} C_{1}\right) \cdot\left(\overline{C_{4}} B_{4} C_{4} C_{3}\right) *\left(C_{2} C_{2}\right) \cdot\left(\overline{C_{4}} B_{4} C_{4}\right) *\left(C_{3} C_{3}\right) \cdot \\
& \cdot\left(\overline{C_{4}} B_{4}\right) *\left(C_{4} C_{4}\right) \cdot\left(\overline{C_{4}} \overline{C_{4}} \overline{C_{3}} \overline{C_{2}}\right) *\left(\overline{C_{1}} \overline{C_{1}}\right) \cdot\left(\overline{C_{4}} \overline{C_{4}} \overline{C_{3}}\right) *\left(\overline{C_{2}} \overline{C_{2}}\right) \cdot \\
& \cdot\left(\overline{C_{4}} \overline{C_{4}}\right) *\left(\overline{C_{3}} \overline{C_{3}}\right) \cdot \overline{C_{4}} *\left(\overline{C_{4}} \overline{C_{4}}\right) \\
& =\left(\overline{C_{4}} B_{4} C_{4} \cdot C_{3} \cdot C_{2}\right) *\left(C_{1} C_{1}\right) \cdot\left(\overline{C_{4}} B_{4} C_{4} \cdot C_{3}\right) *\left(C_{2} C_{2}\right) \cdot \\
& \cdot\left(\overline{C_{4}} B_{4} C_{4}\right) *\left(C_{3} C_{3}\right) \cdot\left(\overline{C_{4}} B_{4} C_{4}\right) *\left(C_{4} C_{4}\right) \cdot\left(\overline{C_{4}} \overline{C_{4}} \cdot \overline{C_{3}} \cdot \overline{C_{2}}\right) *\left(\overline{C_{1}} \overline{C_{1}}\right) . \\
& \cdot\left(\overline{C_{4}} \overline{C_{4}} \cdot \overline{C_{3}}\right) *\left(\overline{C_{2}} \overline{C_{2}}\right) \cdot\left(\overline{C_{4}} \overline{C_{4}}\right) *\left(\overline{C_{3}} \overline{C_{3}}\right) \cdot\left(\overline{C_{4}} \overline{C_{4}}\right)
\end{aligned}
$$

This equation shows that $[[1,1,1,0,1,0, \ldots, 0]] \in G_{g}$. 
Since $\overline{C_{4}} \overline{C_{3}} \overline{C_{6}} \overline{C_{5}} \overline{C_{4}} *[[1,1,1,1,0, \ldots, 0]]=[[1,1,0,0,1,0,1,0, \ldots, 0]]$,

$[[1,1,0,0,1,0,1,0, \ldots, 0]]=\overline{C_{4}} \overline{C_{3}} \overline{C_{6}} \overline{C_{5}} \overline{C_{4}} *\left(B_{4} \overline{B_{4}^{\prime}}\right)$

$=\left(\overline{C_{4}} \overline{C_{3}} \overline{C_{6}} \overline{C_{5}} \overline{C_{4}} B_{4} C_{4} C_{3} C_{2}\right) *\left(C_{1} C_{1}\right) \cdot\left(\overline{C_{4}} \overline{C_{3}} \overline{C_{6}} \overline{C_{5}} \overline{C_{4}} B_{4} C_{4} C_{3}\right) *\left(C_{2} C_{2}\right)$.

$\cdot\left(\overline{C_{4}} \overline{C_{3}} \overline{C_{6}} \overline{C_{5}} \overline{C_{4}} B_{4} C_{4}\right) *\left(C_{3} C_{3}\right) \cdot\left(\overline{C_{4}} \overline{C_{3}} \overline{C_{6}} \overline{C_{5}} \overline{C_{4}} B_{4}\right) *\left(C_{4} C_{4}\right)$.

$\cdot\left(\overline{C_{4}} \overline{C_{3}} \overline{C_{6}} \overline{C_{5}} \overline{C_{4}} \overline{C_{4}} \overline{C_{3}} \overline{C_{2}}\right) *\left(\overline{C_{1}} \overline{C_{1}}\right) \cdot\left(\overline{C_{4}} \overline{C_{3}} \overline{C_{6}} \overline{C_{5}} \overline{C_{4}} \overline{C_{4}} \overline{C_{3}}\right) *\left(\overline{C_{2}} \overline{C_{2}}\right) \cdot$

$\cdot\left(\overline{C_{4}} \overline{C_{3}} \overline{C_{6}} \overline{C_{5}} \overline{C_{4}} \overline{C_{4}}\right) *\left(\overline{C_{3}} \overline{C_{3}}\right) \cdot\left(\overline{C_{4}} \overline{C_{3}} \overline{C_{6}} \overline{C_{5}} \overline{C_{4}}\right) *\left(\overline{C_{4}} \overline{C_{4}}\right)$.

This equation describes $[[1,1,0,0,1,0,1,0, \ldots, 0]]$ as a product of squares of Dehn twists. By using braid relations of $\mathcal{M}_{g}$, we show that these squares of Dehn twists are elements of $G_{g}$ as follows,

$$
\begin{aligned}
&\left(\overline{C_{4}} \overline{C_{3}} \overline{C_{6}} \overline{C_{5}} \overline{C_{4}} B_{4} C_{4} C_{3} C_{2}\right) *\left(C_{1} C_{1}\right) \\
&=\left(\overline{C_{1}} \cdot \overline{C_{4}} B_{4} C_{4} \cdot \overline{C_{2}} \cdot C_{3} \cdot \overline{C_{6}} C_{5} C_{6}\right) *\left(C_{4} C_{4}\right), \\
&\left(\overline{C_{4}} \overline{C_{3}} \overline{C_{6}} \overline{C_{5}} \overline{C_{4}} B_{4} C_{4} C_{3}\right) *\left(C_{2} C_{2}\right) \\
&=\left(\overline{C_{4}} B_{4} C_{4} \cdot \overline{C_{2}} \cdot C_{3} \cdot \overline{C_{6}} C_{5} C_{6}\right) *\left(C_{4} C_{4}\right), \\
&\left(\overline{C_{4}} \overline{C_{3}} \overline{C_{6}} \overline{C_{5}} \overline{C_{4}} B_{4} C_{4}\right) *\left(C_{3} C_{3}\right)=\left(\overline{C_{4}} B_{4} C_{4} \cdot C_{3} \cdot \overline{C_{4}} \overline{C_{4}} \cdot \overline{C_{6}}\right) *\left(C_{5} C_{5}\right) \\
&=\left(\overline{C_{4}} B_{4} C_{4} \cdot C_{3} \cdot \overline{C_{4}} \overline{C_{4}}\right) *\left(\overline{C_{6}} C_{5} C_{6} \cdot \overline{C_{6}} C_{5} C_{6}\right), \\
&\left(\overline{C_{4}} \overline{C_{3}} \overline{C_{6}} \overline{C_{5}} \overline{C_{4}} B_{4}\right) *\left(C_{4} C_{4}\right) \\
&=\left(C_{3} \cdot \overline{C_{4}} B_{4} C_{4} \cdot \overline{C_{6}} C_{5} C_{6} \cdot \overline{C_{4}} \overline{C_{4}}\right) *\left(C_{3} C_{3}\right), \\
&\left(\overline{C_{4}} \overline{C_{3}} \overline{C_{6}} \overline{C_{5}} \overline{C_{4}} \overline{C_{4}} \overline{C_{3}} \overline{C_{2}}\right) *\left(\overline{C_{1}} \overline{C_{1}}\right) \\
&\left(\overline{C_{4}} \overline{C_{3}} \overline{C_{6}} \overline{C_{5}} \overline{C_{4}} \overline{C_{4}} \overline{C_{3}}\right) *\left(\overline{C_{2}} \overline{C_{2}}\right) \\
&=\left(C_{3} \cdot C_{2} \cdot \overline{C_{4}} \overline{C_{4}} \cdot \overline{C_{6}} \overline{C_{5}} C_{6} \cdot \overline{C_{4}} \overline{C_{4}}\right) *\left(C_{3} C_{3}\right), \\
&\left(\overline{C_{4}} \overline{C_{3}} \overline{C_{6}} \overline{C_{5}} \overline{C_{5}} \overline{C_{4}} \overline{C_{4}}\right) *\left(\overline{C_{3}} \overline{C_{3}}\right)=\left(C_{3} \cdot \overline{C_{6}} C_{5} C_{6} \cdot \overline{C_{6}} C_{5} C_{6}\right) *\left(C_{4} C_{4}\right), \\
&\left(\overline{C_{4}} \overline{C_{3}} \overline{C_{6}} \frac{\overline{C_{5}}}{C_{5}} \overline{C_{4}}\right) *\left(\overline{C_{4}} \overline{C_{4}}\right)=\left(C_{3} \cdot \overline{C_{4}} C_{5} C_{4}\right) *\left(C_{6} C_{6}\right) .
\end{aligned}
$$

Lemma 6.12 The odd subchain map $[[1,1,1,0,1,0,1,0,1,0, \ldots, 0]]$ is an element of $G_{g}$.

Proof We can show that this odd subchain map is $G_{g}$-equivalent to $[[0,0,0,0$, $1,1,1,1,1,1,0, \ldots, 0]]$ as follows,

$$
[[1,1,1,0,1,0,1,0,1,0, \ldots, 0]] \underset{\overline{C_{3}}}{\longrightarrow}[[1,1,0,1,1,0,1,0,1,0, \ldots, 0]]
$$




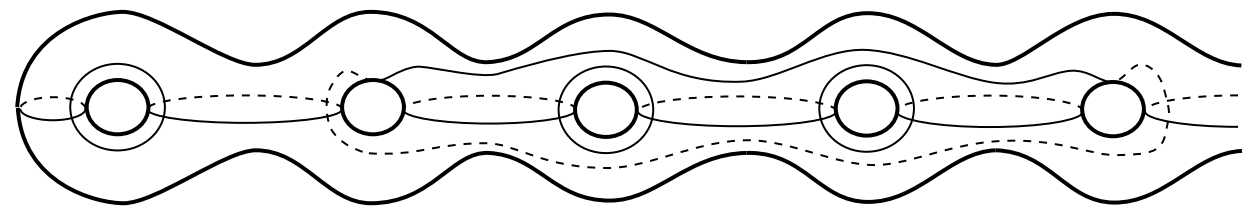

Figure 14

$$
\begin{aligned}
& \underset{\text { Lemma } \underset{[6.8}{\longrightarrow} 2)}{\longrightarrow}[[1,1,0,0,1,1,1,0,1,0, \ldots, 0]] \\
& \underset{C_{3}}{\longrightarrow}[[1,0,0,1,1,1,1,0,1,0, \ldots, 0]] \\
& \underset{\text { Lemma } \underset{[6.8}{\longrightarrow}(2)}{\longrightarrow}[[1,0,0,0,1,1,1,1,1,0, \ldots, 0]] \\
& \overline{C_{3}} \underset{\overline{C_{2}}}{\longrightarrow}[[0,0,0,1,1,1,1,1,1,0, \ldots, 0]] \\
& \underset{\text { Lemma } \underset{6.8]}{\longrightarrow} 2)}{\longrightarrow}[[0,0,0,0,1,1,1,1,1,1,0, \ldots, 0]] .
\end{aligned}
$$

If $g=4,[[0,0,0,0,1,1,1,1,1,1]]=B_{4} \overline{B_{4}^{\prime}}=[[1,1,1,1,0,0,0,0,0,0]]$, which we have already treated in Lemma 6.11] If $g \geq 5$, as we see in Figure 14

$$
[[0,0,0,0,1,1,1,1,1,1,0, \ldots, 0]]=[[1,1,1,1,0,0,0,0,0,0,1, \ldots, 1]],
$$

in the notation of the last odd subchain map, ... is a sequence of 1-tacks. By Lemma 6.8 (2),

$$
[[1,1,1,1,0,0,0,0,0,0,1, \ldots, 1]] \underset{G_{g}}{\widetilde{C}}[[1,1,1,1,1, \ldots, 1,0,0,0,0,0,0]],
$$

which is a product of elements of $G_{g}$ and shorter odd subchain maps.

Therefore, Lemma 6.2 is proved.

\subsection{Step 2 for the case where $g \geq 3$}

Let $\Phi_{2}$ be the natural homomorphism from $\mathcal{M}_{g}$ to $\operatorname{Sp}\left(2 g, \mathbb{Z}_{2}\right)$ defined by the action of $\mathcal{M}_{g}$ on the $\mathbb{Z}_{2}$-coefficient first homology group $H_{1}\left(\Sigma_{g} ; \mathbb{Z}_{2}\right)$. In this section, we will show the following lemma.

Lemma 6.13 $\operatorname{ker} \Phi_{2}$ is a subgroup of $G_{g}$.

We denote the kernel of the natural homomorphism from $\operatorname{Sp}(2 g, \mathbb{Z})$ to $\operatorname{Sp}\left(2 g, \mathbb{Z}_{2}\right)$ by $\mathrm{Sp}^{(2)}(2 g)$. We set a basis of $H_{1}\left(\Sigma_{g} ; \mathbb{Z}\right)$ as in Figure 9 
and define the intersection form $($,$) on H_{1}\left(\Sigma_{g} ; \mathbb{Z}\right)$ to satisfy $\left(x_{i}, y_{j}\right)=\delta_{i, j}$, $\left(x_{i}, x_{j}\right)=\left(y_{i}, y_{j}\right)=0(1 \leq i, j, \leq g)$. An element $a$ of $H_{1}\left(\Sigma_{g} ; \mathbb{Z}\right)$ is called primitive if there is no element $n(\neq 0, \pm 1)$ of $\mathbb{Z}$, and no element $b$ of $H_{1}\left(\Sigma_{g} ; \mathbb{Z}\right)$ such that $a=n b$. For a primitive element $a$ of $H_{1}\left(\Sigma_{g} ; \mathbb{Z}\right)$, we define an isomorphism $T_{a}: H_{1}\left(\Sigma_{g} ; \mathbb{Z}\right) \rightarrow H_{1}\left(\Sigma_{g} ; \mathbb{Z}\right)$ by $T_{a}(v)=v+(a, v) a$. This isomorphism is the action of Dehn twist about a simple closed curve representing $a$ on $H_{1}\left(\Sigma_{g} ; \mathbb{Z}\right)$. We call $T_{a}^{2}$ the square transvection about a. Johnson [15] showed the following result.

Lemma 6.14 $\mathrm{Sp}^{(2)}(2 g)$ is generated by square transvections.

In [11], we showed,

Lemma 6.15 $\mathrm{Sp}^{(2)}(2 g)$ is generated by the square transvections about the primitive elements $\sum_{i=1}^{g}\left(\epsilon_{i} x_{i}+\delta_{i} y_{i}\right)$, where $\epsilon_{i}=0,1$ and $\delta_{i}=0,1$.

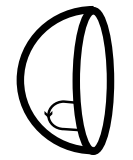

$(-)$

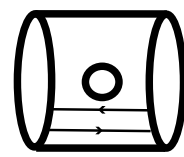

(0)

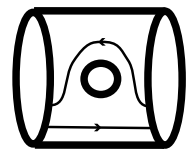

(1)

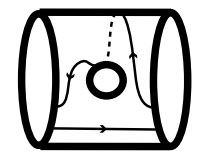

(2)

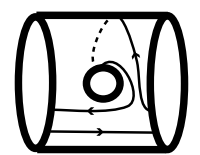

(3)

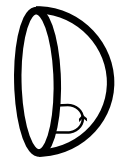

$(+)$

Figure 15

For each element $\left[\left(\epsilon_{1}, \delta_{1}\right), \cdots,\left(\epsilon_{g}, \delta_{g}\right)\right]=\sum_{i=1}^{g}\left(\epsilon_{i} x_{i}+\delta_{i} y_{i}\right)$ (where $\epsilon_{i}=0,1$, $\left.\delta_{i}=0,1\right)$ of $H_{1}\left(\Sigma_{g} ; \mathbb{Z}\right)$, we construct an oriented simple closed curve on $\Sigma_{g}$ which represent this homology class. For each $i$-th block, if $\left(\epsilon_{i}, \delta_{i}\right)=(0,0)$, we prepare (0) of Figure [5] if $\left(\epsilon_{i}, \delta_{i}\right)=(0,1)$, we prepare (1) of Figure [5] if $\left(\epsilon_{i}, \delta_{i}\right)=(1,1)$, we prepare $(2)$ of Figure [15] if $\left(\epsilon_{i}, \delta_{i}\right)=(1,0)$, we prepare $(3)$ of Figure 15. After that, we glue them along the boundaries and cap the left boundary component by (-) of Figure 15] and the right boundary component by $(+)$ of Figure [15] We denote this oriented simple closed curve on $\Sigma_{g}$ by $\left\{\left(\epsilon_{1}, \delta_{1}\right), \cdots,\left(\epsilon_{g}, \delta_{g}\right)\right\}$. Here, we remark that the action of $T_{\left\{\left(\epsilon_{1}, \delta_{1}\right), \cdots,\left(\epsilon_{g}, \delta_{g}\right)\right\}}$ on $H_{1}\left(\Sigma_{g} ; \mathbb{Z}\right)$ equals $T_{\left[\left(\epsilon_{1}, \delta_{1}\right), \cdots,\left(\epsilon_{g}, \delta_{g}\right)\right]}$, and, for any $\phi$ of $\mathcal{M}_{g}, \phi \circ T_{\left\{\left(\epsilon_{1}, \delta_{1}\right), \cdots,\left(\epsilon_{g}, \delta_{g}\right)\right\}} \circ$ $\phi^{-1}=T_{\phi\left(\left\{\left(\epsilon_{1}, \delta_{1}\right), \cdots,\left(\epsilon_{g}, \delta_{g}\right)\right\}\right)}$.

Lemma 6.16 For any $\left\{\left(\epsilon_{1}, \delta_{1}\right), \cdots,\left(\epsilon_{g}, \delta_{g}\right)\right\}$, there is an element $\phi$ of $G_{g}$ such 
that

$$
\begin{aligned}
\phi\left(\left\{\left(\epsilon_{1}, \delta_{1}\right), \cdots,\left(\epsilon_{g}, \delta_{g}\right)\right\}\right) & =\{(0,0),(0,1),(0,0),(0,0), \cdots,(0,0)\} \\
\text { or } & =\{(0,0),(1,1),(0,0),(0,0), \cdots,(0,0)\} \\
\text { or } & =\{(0,0),(0,0),(1,1),(0,0), \cdots,(0,0)\} \\
\text { or } & =\{(0,1),(0,0),(0,0), \cdots,(0,0)\} \\
\text { or } & =\{(1,1),(0,0),(0,0), \cdots,(0,0)\} \\
\text { or } & =\{(0,0),(0,0),(0,0), \cdots,(0,0)\} .
\end{aligned}
$$

(a)

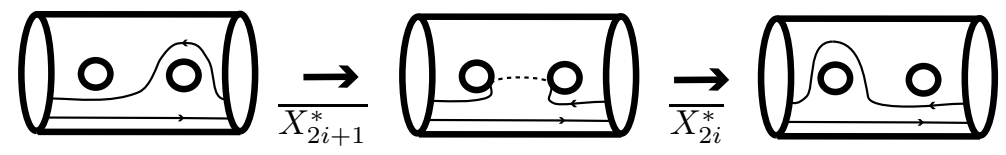

(b)

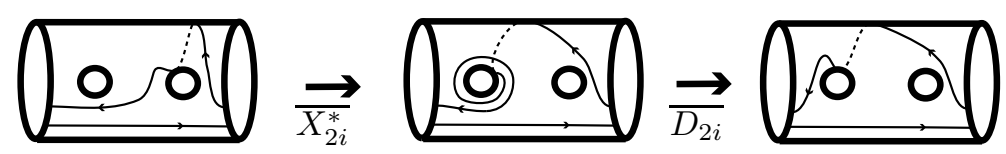

(c)

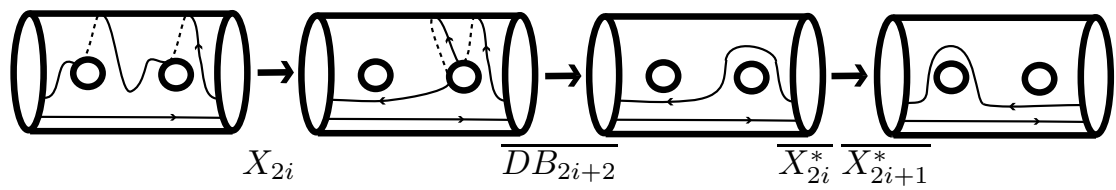

(d)

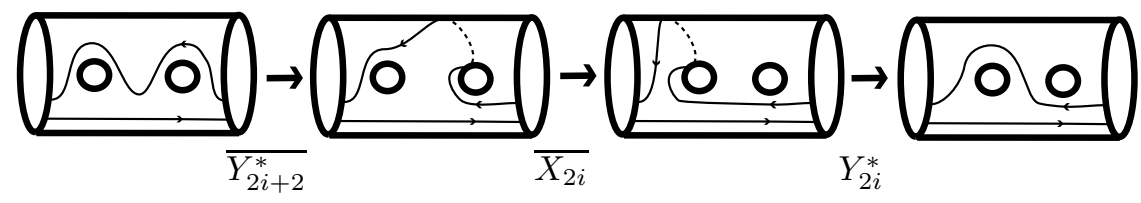

(e)

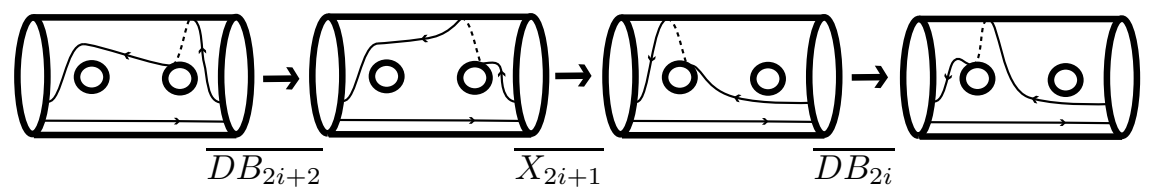

Figure 16

Proof If the $i$-th block is (3), by the action of $\overline{Y_{2 i}}$ if $2 \leq i \leq g-1, C_{2} \overline{C_{1}} \overline{C_{2}}$ if $i=1$, and $C_{2 g} \overline{C_{2 g+1}} \overline{C_{2 g}}$ if $i=g$, this block is changed to (1). Therefore, it suffices to show this lemma in the case where each block is not (3). First we investigate actions of elements of $G_{g}$ on adjacent blocks, say the $i$-th block and 
the $i+1$-st block, where $i \geq 2$. Each picture of Figure 16 shows the action of $G_{g}$ on this adjacent blocks.

$$
\begin{aligned}
& \text { (a) shows }\{\bullet \bullet \bullet,(0,0),(0,1), \bullet \bullet \bullet\} \underset{G_{g}}{\widetilde{\sim}}\{\bullet \bullet \bullet,(0,1),(0,0), \bullet \bullet \bullet\} \text {, } \\
& \text { (b) shows }\{\bullet \bullet \bullet,(0,0),(1,1), \bullet \bullet \bullet\} \underset{G_{g}}{\widetilde{\sim}}\{\bullet \bullet \bullet,(1,1),(0,1), \bullet \bullet \bullet\} \text {, } \\
& \text { (c) shows }\{\bullet \bullet \bullet,(1,1),(1,1), \bullet \bullet \bullet\} \underset{G_{g}}{\widetilde{f}}\{\bullet \bullet \bullet,(0,1),(0,0), \bullet \bullet \bullet\} \text {, } \\
& (d) \text { shows }\{\bullet \bullet \bullet,(0,1),(0,1), \bullet \bullet \bullet\} \widetilde{G_{g}}\{\bullet \bullet \bullet,(0,1),(0,0), \bullet \bullet \bullet\} \text {, } \\
& (e) \text { shows }\{\bullet \bullet \bullet,(0,1),(1,1), \bullet \bullet \bullet\} \underset{G_{g}}{\widetilde{\sim}}\{\bullet \bullet \bullet,(1,1),(0,0), \bullet \bullet \bullet\},
\end{aligned}
$$

where $\bullet \bullet-$ indicates the part which is not changed by the action of $G_{g}$. Let $x=\left\{\left(\epsilon_{1}, \delta_{1}\right), \cdots,\left(\epsilon_{g}, \delta_{g}\right)\right\}$, each of whose block is $(0,0)$ or $(0,1)$ or $(1,1)$. If there are the $j$-th blocks $(1,1)(j \geq 2)$, by (b) and (e), they are gathered to a sequence of $(1,1)$ blocks which begins from the second block. If there are the $j$-th blocks $(0,1)(j \geq 2)$, by (a), they are gathered to a sequence of $(0,1)$ blocks subsequent to the previous sequence of $(1,1)$ blocks. Hence, we showed,

$$
x \underset{G_{g}}{\sim}\left\{\left(\epsilon_{1}, \delta_{1}\right),(1,1), \cdots,(1,1),(0,1), \cdots,(0,1),(0,0), \cdots,(0,0)\right\} .
$$

By (a) and (d), the sequence of $(0,1)$ blocks is altered to $(0,1),(0,0), \cdots,(0,0)$ or $(0,0), \cdots,(0,0)$. By $(\mathrm{c})$, the sequence of $(1,1)$ blocks is altered to $(1,1)$, $(0,1),(0,0), \cdots,(0,1),(0,0)$ (when the length of the sequence is odd) or to $(0,1),(0,0), \cdots,(0,1),(0,0)$ (when the length of the sequence is even). By (a) and $(\mathrm{d}),(1,1),(0,1),(0,0), \cdots,(0,1),(0,0)$ is altered to $(1,1),(0,1),(0,0), \cdots$, $(0,0),(0,0)$ or $(1,1),(0,0),(0,0), \cdots,(0,0),(0,0)$, and $(0,1),(0,0), \cdots,(0,1)$, $(0,0)$ to $(0,1),(0,0), \cdots,(0,0),(0,0)$ or $(0,0),(0,0), \cdots,(0,0),(0,0)$. Therefore, we showed,

$$
\begin{aligned}
& x \underset{G_{g}}{\sim}\left\{\left(\epsilon_{1}, \delta_{1}\right),(1,1),(0,0),(0,0), \cdots,(0,0),(0,0),(0,0), \cdots,(0,0)\right\}, \\
& \text { or } \underset{G_{g}}{\sim}\left\{\left(\epsilon_{1}, \delta_{1}\right),(1,1),(0,0),(0,0), \cdots,(0,0),(0,1),(0,0), \cdots,(0,0)\right\} \text {, } \\
& \text { or } \underset{G_{g}}{\sim}\left\{\left(\epsilon_{1}, \delta_{1}\right),(1,1),(0,1),(0,0), \cdots,(0,0),(0,0),(0,0), \cdots,(0,0)\right\} \text {, } \\
& \text { or } \underset{G_{g}}{\sim}\left\{\left(\epsilon_{1}, \delta_{1}\right),(1,1),(0,1),(0,0), \cdots,(0,0),(0,1),(0,0), \cdots,(0,0)\right\} \text {, } \\
& \text { or } \underset{G_{g}}{\sim}\left\{\left(\epsilon_{1}, \delta_{1}\right),(0,1),(0,0),(0,0), \cdots,(0,0),(0,0),(0,0), \cdots,(0,0)\right\} \text {, } \\
& \text { or } \underset{G_{g}}{\sim}\left\{\left(\epsilon_{1}, \delta_{1}\right),(0,1),(0,0),(0,0), \cdots,(0,0),(0,1),(0,0), \cdots,(0,0)\right\} \text {, } \\
& \text { or } \underset{G_{g}}{\widetilde{2}}\left\{\left(\epsilon_{1}, \delta_{1}\right),(0,0),(0,0),(0,0), \cdots,(0,0),(0,0),(0,0), \cdots,(0,0)\right\} \text {, }
\end{aligned}
$$




$$
\text { or } \underset{G_{g}}{\sim}\left\{\left(\epsilon_{1}, \delta_{1}\right),(0,0),(0,0),(0,0), \cdots,(0,0),(0,1),(0,0), \cdots,(0,0)\right\} .
$$

In the second case,

$$
\begin{aligned}
& \left\{\left(\epsilon_{1}, \delta_{1}\right),(1,1),(0,0), \cdots,(0,0),(0,1),(0,0), \cdots,(0,0)\right\} \\
& \widetilde{G_{g}}\left\{\left(\epsilon_{1}, \delta_{1}\right),(1,1),(0,1),(0,0), \cdots,(0,0)\right\}(\text { by }(a)) .
\end{aligned}
$$

In the 4-th case,

$$
\begin{aligned}
& \left\{\left(\epsilon_{1}, \delta_{1}\right),(1,1),(0,1),(0,0), \cdots,(0,0),(0,1),(0,0), \cdots,(0,0)\right\} \\
& \underset{G_{g}}{\sim}\left\{\left(\epsilon_{1}, \delta_{1}\right),(1,1),(0,1),(0,1),(0,0), \cdots,(0,0)\right\}(\text { by }(a)) \\
& \underset{G_{g}}{\sim}\left\{\left(\epsilon_{1}, \delta_{1}\right),(1,1),(0,1),(0,0),(0,0), \cdots,(0,0)\right\}(\text { by }(d)) .
\end{aligned}
$$

In the 6 -th case,

$$
\begin{aligned}
& \left\{\left(\epsilon_{1}, \delta_{1}\right),(0,1),(0,0), \cdots,(0,0),(0,1),(0,0), \cdots,(0,0)\right\} \\
& \widetilde{G_{g}}\left\{\left(\epsilon_{1}, \delta_{1}\right),(0,1),(0,1),(0,0), \cdots,(0,0)\right\}(\text { by }(a)) \\
& \widetilde{G_{g}}\left\{\left(\epsilon_{1}, \delta_{1}\right),(0,1),(0,0),(0,0), \cdots,(0,0)\right\}(\text { by }(d)) .
\end{aligned}
$$

In the 8-th case,

$$
\begin{aligned}
& \left\{\left(\epsilon_{1}, \delta_{1}\right),(0,0), \cdots,(0,0),(0,1),(0,0), \cdots,(0,0)\right\} \\
& \widetilde{G_{g}}\left\{\left(\epsilon_{1}, \delta_{1}\right),(0,1),(0,0), \cdots,(0,0)\right\}(\text { by }(a)) .
\end{aligned}
$$

Therefore,

$$
\begin{aligned}
& x \underset{G_{g}}{\widetilde{a}}\left\{\left(\epsilon_{1}, \delta_{1}\right),(1,1),(0,0),(0,0), \cdots,(0,0)\right\}, \\
& \text { or } \underset{G_{g}}{\sim}\left\{\left(\epsilon_{1}, \delta_{1}\right),(1,1),(0,1),(0,0), \cdots,(0,0)\right\} \text {, } \\
& \text { or } \underset{G_{g}}{\sim}\left\{\left(\epsilon_{1}, \delta_{1}\right),(0,1),(0,0),(0,0), \cdots,(0,0)\right\} \text {, } \\
& \text { or } \underset{G_{g}}{\sim}\left\{\left(\epsilon_{1}, \delta_{1}\right),(0,0),(0,0),(0,0), \cdots,(0,0)\right\} \text {. }
\end{aligned}
$$

There are 7 cases remained to consider,

$$
\begin{array}{ll}
\{(0,0),(1,1),(0,1),(0,0), \cdots,(0,0)\}, & \{(0,1),(1,1),(0,0),(0,0), \cdots,(0,0)\}, \\
\{(0,1),(1,1),(0,1),(0,0), \cdots,(0,0)\}, & \{(0,1),(0,1),(0,0),(0,0), \cdots,(0,0)\}, \\
\{(1,1),(1,1),(0,0),(0,0), \cdots,(0,0)\}, & \{(1,1),(1,1),(0,1),(0,0), \cdots,(0,0)\}, \\
\{(1,1),(0,1),(0,0),(0,0), \cdots,(0,0)\} . &
\end{array}
$$


By (b), the first one is $G_{g}$-equivalent to $\{(0,0),(0,0),(1,1),(0,0), \cdots,(0,0)\}$. Here, we observe actions of $G_{g}$ on the first and the second blocks,

$$
\begin{aligned}
& \{(0,1),(1,1), \cdots\} \underset{C_{1}}{\longrightarrow}\{(1,1),(1,1), \cdots\} \underset{D_{B_{4}} \cdot C_{3} \cdot C_{2}}{\longrightarrow}\{(0,0),(0,1), \cdots\}, \\
& \{(0,1),(0,1), \cdots\} \underset{C_{1}}{\longrightarrow}\{(1,1),(0,1), \cdots\} \underset{C_{3} C_{2}}{\longrightarrow}\{(0,0),(1,1), \cdots\} .
\end{aligned}
$$

By the above observation, we see,

$$
\begin{aligned}
& \{(0,1),(1,1),(0,0), \cdots,(0,0)\} \underset{G_{g}}{\widetilde{f}}\{(1,1),(1,1),(0,0), \cdots,(0,0)\} \\
& \widetilde{G_{g}}\{(0,0),(0,1),(0,0), \cdots,(0,0)\}, \\
& \{(0,1),(1,1),(0,1), \cdots,(0,0)\} \underset{G_{g}}{\widetilde{f}}\{(1,1),(1,1),(0,1), \cdots,(0,0)\} \\
& \widetilde{G_{g}}\{(0,0),(0,1),(0,1), \cdots,(0,0)\} \\
& \widetilde{G_{g}}\{(0,0),(0,1),(0,0), \cdots,(0,0)\}, \\
& \{(0,1),(0,1),(0,0), \cdots,(0,0)\} \underset{G_{g}}{\widetilde{\sim}}\{(1,1),(0,1),(0,0), \cdots,(0,0)\} \\
& \widetilde{G_{g}}\{(0,0),(1,1),(0,0), \cdots,(0,0)\} .
\end{aligned}
$$

Hence, we showed that any $x$ is $G_{g}$-equivalent to the elements listed in the statement of this Lemma.

Since

$$
\begin{aligned}
& T_{\{(0,1),(0,0), \cdots,(0,0)\}}^{2}=D_{2}, \quad T_{\{(1,1),(0,0), \cdots,(0,0)\}}^{2}=\left(C_{1} C_{2} C_{1}^{-1}\right)^{2}, \\
& T_{\{(0,0),(1,1),(0,0), \cdots,(0,0)\}}^{2}=\left(Y_{2}^{*}\right)^{2}, \quad T_{\{(0,0),(0,1),(0,0), \cdots,(0,0)\}}^{2}=D_{4}, \\
& T_{\{(0,0),(0,0),(1,1),(0,0), \cdots,(0,0)\}}^{2}=\left(Y_{4}^{*}\right)^{2}, \quad T_{\{(0,0), \cdots,(0,0)\}}^{2}=i d,
\end{aligned}
$$

these are elements of $G_{g}$. By this fact and Lemma 6.2. Lemma 6.13 is proved.

\section{3 $\quad$ Step 3 for the case where $g \geq 3$}

As in the previous subsection, let $\Phi_{2}: \mathcal{M}_{g} \rightarrow \operatorname{Sp}\left(2 g, \mathbb{Z}_{2}\right)$ be the natural homomorphism. Let $q_{1}: H_{1}\left(\Sigma_{g} ; \mathbb{Z}_{2}\right) \rightarrow \mathbb{Z}_{2}$ be the quadratic form associated with the intersection form $(,)_{2}$ of $H_{1}\left(\Sigma_{g} ; \mathbb{Z}_{2}\right)$ which satisfies, for the basis $x_{i}, y_{i}$ of $H_{1}\left(\Sigma_{g} ; \mathbb{Z}_{2}\right)$ indicated on Figure 9 $q_{1}\left(x_{1}\right)=q_{1}\left(y_{1}\right)=1$, and $q_{1}\left(x_{i}\right)=q_{1}\left(y_{i}\right)=0$ when $i \neq 1$. We define $\mathrm{O}_{q_{1}}\left(2 g, \mathbb{Z}_{2}\right)=\left\{\phi \in \operatorname{Aut}\left(H_{1}\left(\Sigma_{g} ; \mathbb{Z}_{2}\right)\right) \mid q_{1}(\phi(x))=\right.$ $q_{1}(x)$ for any $\left.x \in H_{1}\left(\Sigma_{g} ; \mathbb{Z}_{2}\right)\right\}$, then $\mathcal{S P}_{g}\left[q_{1}\right]=\Phi_{2}^{-1}\left(\mathrm{O}_{q_{1}}\left(2 g, \mathbb{Z}_{2}\right)\right)$. Because of Lemma 6.13] if we show $\Phi_{2}\left(G_{g}\right)=\mathrm{O}_{q_{1}}\left(2 g, \mathbb{Z}_{2}\right)$, then $G_{g}=\mathcal{S P}_{g}\left[q_{1}\right]$ follows. 
For any $z \in H_{1}\left(\Sigma_{g} ; \mathbb{Z}_{2}\right)$ such that $q_{1}(z)=1$, we define $\mathbb{T}_{z}(x)=x+(z, x)_{2} z$. Then $\mathbb{T}_{z}$ is an element of $\mathrm{O}_{q_{1}}\left(2 g, \mathbb{Z}_{2}\right)$, and we call this a $\mathbb{Z}_{2}$-transvection about $z$. Dieudonné [4] showed the following Theorem (see also [7, Chap.14]).

Theorem 6.17 4, Proposition 14 on p.42] When $g \geq 3, \mathrm{O}_{q_{1}}\left(2 g, \mathbb{Z}_{2}\right)$ is generated by $\mathbb{Z}_{2}$-transvections.

Let $\Lambda_{g}$ be the set of $z$ of $H_{1}\left(\Sigma_{g} ; \mathbb{Z}_{2}\right)$ such that $q(z)=1$. For any elements $z_{1}$ and $z_{2}$ of $\Lambda_{g}$, we define $z_{1} \square z_{2}=z_{1}+\left(z_{2}, z_{1}\right)_{2} z_{2}$. Here, we remark that $\mathbb{T}_{z_{1}}^{2}=\mathrm{id}, \mathbb{T}_{z_{2}} \mathbb{T}_{z_{1}} \mathbb{T}_{z_{2}}^{-1}=\mathbb{T}_{z_{1} \square z_{2}}$ and $\left(z_{1} \square z_{2}\right) \square z_{2}=z_{1}$. An element $\epsilon_{1} x_{1}+$ $\delta_{1} y_{1}+\cdots+\epsilon_{g} x_{g}+\delta_{g} y_{g}$ of $H_{1}\left(\Sigma_{g} ; \mathbb{Z}_{2}\right)$ is denoted by $\left[\left(\epsilon_{1}, \delta_{1}\right), \cdots,\left(\epsilon_{g}, \delta_{g}\right)\right]$, and each $\left(\epsilon_{i}, \delta_{i}\right)$ is called the $i$-th block. We remark that $q\left(\left[\left(\epsilon_{1}, \delta_{1}\right), \cdots,\left(\epsilon_{g}, \delta_{g}\right)\right]\right)=$ $\left(\epsilon_{1}+\delta_{1}+\epsilon_{1} \delta_{1}\right)+\epsilon_{2} \delta_{2}+\cdots \epsilon_{g} \delta_{g}$.

Lemma 6.18 Under the operation $\square, \Lambda_{g}$ is generated by $x_{1}, y_{1}, x_{1}+x_{2}$, $x_{i}+y_{i}(2 \leq i \leq g), x_{i}+y_{i}+x_{i+1}(2 \leq i \leq g-1)$, and $x_{i}+x_{i+1}+y_{i+1}$ $(2 \leq i \leq g-1)$.

Proof For an element $\left[\left(\epsilon_{1}, \delta_{1}\right), \cdots,\left(\epsilon_{g}, \delta_{g}\right)\right]$ of $H_{1}\left(\Sigma_{g} ; \mathbb{Z}_{2}\right)$, let the $j$-th block be the right most block which is $(1,1)$. When $j \geq 3$, there exist 4 cases of the combination of the $(j-1)$-st block and the $j$-th block: $[\cdots,(1,1),(1,1), \cdots]$, $[\cdots,(0,0),(1,1), \cdots],[\cdots,(0,1),(1,1), \cdots],[\cdots,(1,0),(1,1), \cdots]$. In each case, we can reduce $j$ at least 1 . In fact,

$$
\begin{gathered}
{[\cdots,(1,1),(1,1), \cdots] \square\left(x_{j-1}+x_{j}+y_{j}\right)=[\cdots,(0,1),(0,0), \cdots],} \\
{[\cdots,(0,0),(1,1), \cdots] \square\left(x_{j-1}+y_{j-1}+x_{j}\right)=[\cdots,(1,1),(0,1), \cdots],} \\
{[\cdots,(0,1),(1,1), \cdots] \square\left(x_{j-1}+x_{j}+y_{j}\right)=[\cdots,(1,1),(0,0), \cdots],} \\
\left([\cdots,(1,0),(1,1), \cdots] \square\left(x_{j-1}+y_{j-1}\right)\right) \square\left(x_{j-1}+x_{j}+y_{j}\right) \\
=[\cdots,(1,1),(0,0), \cdots] .
\end{gathered}
$$

When $j=2$, since $q\left(\left[\left(\epsilon_{1}, \delta_{1}\right), \cdots,\left(\epsilon_{g}, \delta_{g}\right)\right]\right)=1,\left[\left(\epsilon_{1}, \delta_{1}\right), \cdots,\left(\epsilon_{g}, \delta_{g}\right)\right]$ must be $[(0,0),(1,1), \cdots]$. Because of an equation

$$
\left([(0,0),(1,1), \cdots] \square\left(x_{1}+x_{2}\right)\right) \square y_{1}=[(1,1),(0,1), \cdots],
$$

we can reduce $j$ to 1 . When $j=1$, if every $i$-th $(i \geq 2)$ block is $(0,0)$, then it is $x_{1}+y_{1}$, which is equal to $x_{1} \square y_{1}$. If there exist at least one of the $i$-th 
$(i \geq 2)$ blocks which are $(1,0)$ or $(0,1)$, then,

$$
\begin{aligned}
& {[\cdots,(0,0),(1,0), \cdots] \square\left(x_{i-1}+x_{i}+y_{i}\right)=[\cdots,(1,0),(0,1), \cdots],} \\
& {[\cdots,(1,0),(0,0), \cdots] \square\left(x_{i-1}+y_{i-1}+x_{i}\right)=[\cdots,(0,1),(1,0), \cdots],} \\
& {[\cdots,(0,0),(0,1), \cdots] \square\left(x_{i-1}+x_{i}+y_{i}\right)=[\cdots,(1,0),(1,0), \cdots],} \\
& {[\cdots,(0,1),(0,0), \cdots] \square\left(x_{i-1}+y_{i-1}+x_{i}\right)=[\cdots,(1,0),(1,0), \cdots] .}
\end{aligned}
$$

Therefore, we can alter this to an element, each $i$-th $(i \geq 2)$ block of which is $(1,0)$ or $(0,1)$. If the $i$-th block of this is $(0,1)$, then

$$
[\cdots,(0,1), \cdots] \square\left(x_{i}+y_{i}\right)=[\cdots,(1,0), \cdots] .
$$

Therefore, it suffices to consider the case where the first block is $(1,1)$ and other blocks are $(1,0)$. In this case,

$$
\left([\cdots,(1,0),(1,0)] \square\left(x_{g-1}+y_{g-1}+x_{g}\right)\right) \square\left(x_{g-1}+y_{g-1}\right)=[\cdots,(1,0),(0,0)] .
$$

By applying the same operation repeatedly, we get $[(1,1),(1,0),(0,0), \cdots$, $(0,0)]$, which is equal to $y_{1} \square\left(x_{1}+x_{2}\right)$.

This lemma and Theorem 6.17 shows that

Corollary 6.19 $\mathrm{O}_{q_{1}}\left(2 g, \mathbb{Z}_{2}\right)$ is generated by $\mathbb{T}_{x_{1}}, \mathbb{T}_{y_{1}}, \mathbb{T}_{x_{1}+x_{2}}, \mathbb{T}_{x_{i}+y_{i}}(2 \leq$ $i \leq g), \mathbb{T}_{x_{i}+y_{i}+x_{i+1}}(2 \leq i \leq g-1)$, and $\mathbb{T}_{x_{i}+x_{i+1}+y_{i+1}}(2 \leq i \leq g-1)$.

Since $G_{g}$ is a subgroup of $\mathcal{S P}_{g}\left[q_{1}\right], \Phi_{2}\left(G_{g}\right) \subset \mathrm{O}_{q_{1}}\left(2 g, \mathbb{Z}_{2}\right)$. On the other hand, the fact that $\Phi_{2}\left(C_{1}\right)=\mathbb{T}_{x_{1}}, \Phi_{2}\left(C_{2}\right)=\mathbb{T}_{y_{1}}, \Phi_{2}\left(C_{3}\right)=\mathbb{T}_{x_{1}+x_{2}}, \Phi_{2}\left(X_{2 i}\right)=$ $\mathbb{T}_{x_{i}+y_{i}+x_{i+1}}(2 \leq i \leq g-1), \Phi_{2}\left(X_{2 i+1}\right)=\mathbb{T}_{x_{i}+x_{i+1}+y_{i+1}}(2 \leq i \leq g-1)$, $\Phi_{2}\left(Y_{2 j}\right)=\mathbb{T}_{x_{j}+y_{j}}(2 \leq j \leq g-1), \Phi_{2}\left(X_{2 g}\right)=\mathbb{T}_{x_{g}+y_{g}}$, and Corollary 6.19] show $\Phi_{2}\left(G_{g}\right) \supset \mathrm{O}_{q_{1}}\left(2 g, \mathbb{Z}_{2}\right)$. Therefore we proved that $\mathcal{S} \mathcal{P}_{g}\left[q_{1}\right]=G_{g}$ when $g \geq 3$.

\subsection{Genus 2 case: Reidemeister-Schreier method}

Birman and Hilden showed the following Theorem.

Theorem 6.20 [2] $\mathcal{M}_{2}$ is generated by $C_{1}, C_{2}, C_{3}, C_{4}, C_{5}$ and its defining relations are:

(1) $C_{i} C_{j}=C_{j} C_{i}$, if $|i-j| \geq 2, i, j=1,2,3,4,5$,

(2) $C_{i} C_{i+1} C_{i}=C_{i+1} C_{i} C_{i+1}, i=1,2,3,4$, 
(3) $\left(C_{1} C_{2} C_{3} C_{4} C_{5}\right)^{6}=1$,

(4) $\left(C_{1} C_{2} C_{3} C_{4} C_{5} C_{5} C_{4} C_{3} C_{2} C_{1}\right)^{2}=1$,

(5) $C_{1} C_{2} C_{3} C_{4} C_{5} C_{5} C_{4} C_{3} C_{2} C_{1} \rightleftarrows C_{i}, i=1,2,3,4,5$,

where $\rightleftarrows$ means "commute with".

We call (1) (2) of the above relations braid relations. We will use the wellknown method, called the Reidemeister-Schreier method [18, §2.3], to show $\mathcal{S} \mathcal{P}_{2}\left[q_{1}\right] \subset G_{2}$. We review (a part of) this method.

Let $G$ be a group generated by finite elements $g_{1}, \ldots, g_{m}$ and $H$ be a finite index subgroup of $G$. For two elements $a, b$ of $G$, we write $a \equiv b \bmod H$ if there is an element $h$ of $H$ such that $a=h b$. A finite subset $S$ of $G$ is called a coset representative system for $G \bmod H$, if, for each elements $g$ of $G$, there is only one element $\overline{\bar{g}} \in S$ such that $g \equiv \overline{\bar{g}} \bmod H$. The set $\left\{s g_{i}{\overline{\overline{s g_{i}}}}^{-1} \mid i=1, \ldots, m, s \in S\right\}$ generates $H$.

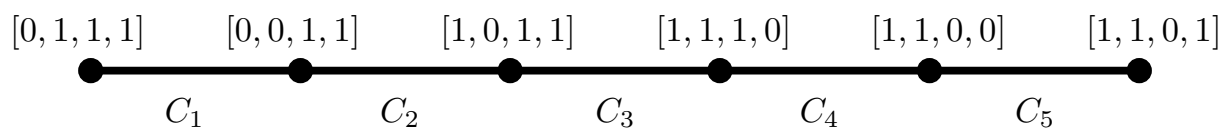

Figure 17

For the sake of giving a coset representative system for $\mathcal{M}_{2}$ modulo $\mathcal{S} \mathcal{P}_{2}\left[q_{1}\right]$, we will draw a graph $\Gamma$ which represents the action of $\mathcal{M}_{2}$ on the quadratic forms of $H_{1}\left(\Sigma_{2} ; \mathbb{Z}_{2}\right)$ with Arf invariants 1 . Let $\left[\epsilon_{1}, \epsilon_{2}, \epsilon_{3}, \epsilon_{4}\right]$ denote the quadratic form $q^{\prime}$ of $H_{1}\left(\Sigma_{2} ; \mathbb{Z}_{2}\right)$ such that $q^{\prime}\left(x_{1}\right)=\epsilon_{1}, q^{\prime}\left(y_{1}\right)=\epsilon_{2}, q^{\prime}\left(x_{2}\right)=\epsilon_{3}, q^{\prime}\left(y_{2}\right)=\epsilon_{4}$. Each vertex of $\Gamma$ corresponds to a quadratic form. For each generator $C_{i}$ of $\mathcal{M}_{2}$, we denote its action on $H_{1}\left(\Sigma_{2} ; \mathbb{Z}_{2}\right)$ by $\left(C_{i}\right)_{*}$. For the quadratic form $q^{\prime}$ indicated by the symbol $\left[\epsilon_{1}, \epsilon_{2}, \epsilon_{3}, \epsilon_{4}\right]$, let $\delta_{1}=q^{\prime}\left(\left(C_{i}\right)_{*} x_{1}\right), \delta_{2}=q^{\prime}\left(\left(C_{i}\right)_{*} y_{1}\right)$, $\delta_{3}=q^{\prime}\left(\left(C_{i}\right)_{*} x_{2}\right)$, and $\delta_{4}=q^{\prime}\left(\left(C_{i}\right)_{*} y_{2}\right)$. Then, we connect two vertices, corresponding to $\left[\epsilon_{1}, \epsilon_{2}, \epsilon_{3}, \epsilon_{4}\right],\left[\delta_{1}, \delta_{2}, \delta_{3}, \delta_{4}\right]$ respectively, by the edge with the letter $C_{i}$. We remark that this action is a right action. For simplicity, we omit the edge whose ends are the same vertex. As a result, we get a graph $\Gamma$ as in Figure 17. The words $S=\left\{1, C_{5}, C_{4}, C_{4} C_{3}, C_{4} C_{3} C_{2}, C_{4} C_{3} C_{2} C_{1}\right\}$, which correspond to the edge paths beginning from $[1,1,0,0]$ on $\Gamma$, define a coset representative system for $\mathcal{M}_{2}$ modulo $\mathcal{S P}_{2}\left[q_{1}\right]$. For each element $g$ of $\mathcal{M}_{2}$, we can give a $\overline{\bar{g}} \in S$ with using this graph. For example, say $g=C_{2} C_{4} C_{5} C_{3}$, we follow an edge path assigned to this word which begins from $[1,1,0,0]$, (note that we read words from left to right) then we arrive at the vertex 
Table 1: Generators of $\mathcal{S P}_{2}\left[q_{1}\right]$

\begin{tabular}{c|cccc} 
& \multicolumn{1}{|c}{$C_{1}$} & $C_{2}$ & $C_{3}$ \\
\hline 1 & 1 & 1 & 1 \\
$C_{5}$ & $C_{1}$ & $C_{2}$ & $C_{3}$ \\
$C_{4}$ & $C_{1}$ & $C_{2}$ & 1 \\
$C_{4} C_{3}$ & $C_{1}$ & 1 & $C_{3}^{-1} D_{4} C_{3}$ \\
$C_{4} C_{3} C_{2}$ & 1 & & $C_{2}^{-1} C_{3}^{-1} D_{4} C_{3} C_{2}$ & $C_{2}$ \\
$C_{4} C_{3} C_{2} C_{1}$ & $C_{1}^{-1} C_{2}^{-1} C_{3}^{-1} D_{4} C_{3} C_{2} C_{1}$ & $C_{1}$ & $C_{2}$ \\
& \multicolumn{2}{c}{$C_{4}$} & $C_{5}$ & \\
& & 1 & 1 & \\
& $C_{5}$ & 1 & $D_{5}$ & \\
& $C_{4}$ & $D_{4}$ & $D_{5}^{-1} X_{4} D_{5}$ & \\
& $C_{4} C_{3}$ & $C_{3}$ & $D_{5}^{-1} X_{4} D_{5}$ & \\
& $C_{4} C_{3} C_{2}$ & $C_{3}$ & $D_{5}^{-1} X_{4} D_{5}$ & \\
& $C_{4} C_{3} C_{2} C_{1}$ & $C_{3}$ & $D_{5}^{-1} X_{4} D_{5}$ & \\
& & & &
\end{tabular}

$[1,0,1,1]$. The element in $T$ which begins from $[1,1,0,0]$ and ends at $[1,0,1,1]$ is $C_{4} C_{3}$. Hence, $\overline{\overline{C_{2} C_{4} C_{5} C_{3}}}=C_{4} C_{3}$. We list in Table 1 the set of generators $\left\{s C_{i}{\overline{\overline{s C_{i}}}}^{-1} \mid i=1, \ldots, 5, s \in S\right\}$ of $\mathcal{S P}_{g}\left[q_{1}\right]$. In Table 1 vertical direction is a coset representative system $S$, horizontal direction is a set of generators $\left\{C_{1}, C_{2}, C_{3}, C_{4}, C_{5}\right\}$. We can check this table by Figure 17 and braid relations. For example,

$$
\begin{aligned}
& C_{4} C_{3} C_{2} C_{1} \cdot C_{2}{\overline{\bar{C}_{4} C_{3} C_{2} C_{1} \cdot C_{2}}}^{-1}=C_{4} C_{3} C_{2} C_{1} C_{2}\left(C_{4} C_{3} C_{2} C_{1}\right)^{-1} \\
& =C_{4} C_{3} C_{2} C_{1} C_{2} C_{1}^{-1} C_{2}^{-1} C_{3}^{-1} C_{4}^{-1}=C_{4} C_{3} C_{2} C_{2}^{-1} C_{1} C_{2} C_{2}^{-1} C_{3}^{-1} C_{4}^{-1} \\
& =C_{4} C_{3} C_{1} C_{3}^{-1} C_{4}^{-1}=C_{1} .
\end{aligned}
$$

This table shows that $\mathcal{S P}_{2}\left[q_{1}\right] \subset G_{2}$.

\section{$7 \quad$ Proof of Theorem 5.1}

We embed $H_{g-1}$ standardly in $S^{3}=\partial D_{4}$ such that there is a 2-sphere separating $F_{3,3}$ and $H_{g-1}$, and make a connected sum $F_{3,3} \# \partial H_{g-1}$ as indicated in Figure 18. Then, we can see $\left(\mathbb{C P}^{2}, K_{3} \# \Sigma_{g-1}\right)=\left(\mathbb{C P}^{2},\left(F_{3,3} \# \partial H_{g-1}\right) \cup D_{3}\right)$, where $K_{3}$ is the non-singular plane curve of degree 3 and $D_{3}$ is parallel three disks which is used to construct $K_{3}$ in 4 We identify $K_{3} \# \Sigma_{g-1}$ with $\Sigma_{g}$ so that 


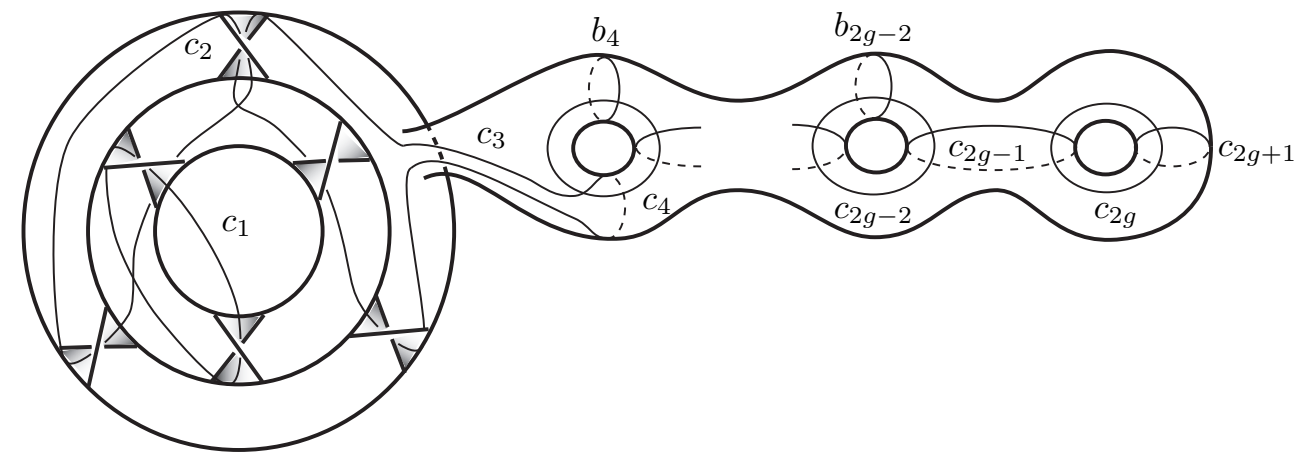

Figure 18

simple closed curves with the same symbol are identified. Then $q_{K_{3} \# \Sigma_{g-1}}=q_{1}$. We will show that each elements of $\mathcal{S P}_{g}\left[q_{K_{3} \# \Sigma_{g-1}}\right]=\mathcal{S P}_{g}\left[q_{1}\right]$ is extendable.

Each regular neighborhood of $c_{1}, c_{2}, c_{3}, C_{i+1}\left(c_{i}\right)(4 \leq i \leq 2 g)$, and $C_{2 j}\left(b_{2 j}\right)$ $(2 \leq j \leq g-1)$ is Hopf band. Therefore, by Proposition 2.1] $C_{1}, C_{2}, C_{3}$, $C_{i+1} C_{i} \overline{C_{i+1}} \quad(4 \leq i \leq 2 g)$, and $C_{2 j} B_{2 j} \overline{C_{2 j}} \quad(2 \leq j \leq g-1)$ are elements of $\mathcal{E}\left(\mathbb{C P}^{2}, K_{3} \# \Sigma_{g-1}\right)$. Each regular neighborhood of $c_{i}(4 \leq i \leq 2 g+1), b_{2 j}$ $(2 \leq i \leq g-1)$ is an annulus standardly embedded in $S^{3}=\partial D^{4}$. We can deform this annulus as indicated in Figure 1]. Therefore, $C_{i}^{2}(4 \leq i \leq 2 g+1), B_{2 j}^{2}$ $(2 \leq j \leq g-1)$ are elements of $\mathcal{E}\left(\mathbb{C P}^{2}, K_{3} \# \Sigma_{g-1}\right)$. Finally, the extendability of $B_{4} C_{5} C_{7} \ldots C_{2 g+1}$ follows from the proof of Lemma 2.2 in 11]. Therefore, we showed $\mathcal{S} \mathcal{P}_{g}\left[q_{K_{3} \# \Sigma_{g-1}}\right] \subset \mathcal{E}\left(\mathbb{C P}^{2}, K_{3} \# \Sigma_{g-1}\right)$. On the other hand, by the definition of the Rokhlin quadratic form $q_{K_{3} \# \Sigma_{g-1}}$, we see $\mathcal{E}\left(\mathbb{C P}^{2}, K_{3} \# \Sigma_{g-1}\right)$ $\subset \mathcal{S P} \mathcal{P}_{g}\left[q_{K_{3} \# \Sigma_{g-1}}\right]$. Theorem 5.1 follows.

\section{Acknowledgments}

The author would like to express his gratitude to Professors Masaharu Ishikawa, Masahico Saito, and Akira Yasuhara for fruitful discussions and comments. The author would also like to thank the referee, whose comments and corrections improved the paper. This research was partially supported by Grant-in-Aid for Encouragement of Young Scientists (No. 16740038), Ministry of Education, Culture, Sports, Science and Technology, Japan. 


\section{References}

[1] S. Akbulut and R. Kirby, Branched covers of surfaces in 4-manifolds, Math. Ann. 252 (1980), 111-131. MathReview

[2] J.S. Birman and H. Hilden, On the mapping class group of closed surface as covering spaces, In "Advances in the theory of Riemann surfaces" Ann. of Math. Studies 66 (1971), 81-115. MathReview

[3] J. Boyle, Classifying 1-handles attached to knotted surfaces, Trans. Amer. Math. Soc. 306 (1988), 475-487. MathReview

[4] J. Dieudonné, Sur les groupes classiques, Actualites Sci. Ind., no. $1040=$ Publ. Inst. Math. Univ. Strasbourg (N.S.) no. 1 (1945). Hermann et Cie., Paris, 1948. MathReview

[5] M. Freedman and R. Kirby, A geometric proof of Rochlin's theorem, Proc. Symp. Pure Math. 32 (1978), 85-97. MathReview

[6] R. Gompf and A. Stipsicz, 4-manifolds and Kirby calculus, Grad. Stud. in Math. 20, American Mathematical Society, 1999. MathReview

[7] L.C. Grove, Classical groups and geometric algebra, Grad. Stud. in Math. 39, American Mathematical Society, 2002. MathReview

[8] J.L. Harer, Stability of the homology of the moduli spaces of Riemann surfaces with spin structure, Math. Ann. 287 (1990), 323-334. MathReview

[9] J.L. Harer, The rational Picard group of the moduli space of Riemann surfaces with spin structure, Contemp. Math. 150 (1993), 107-136. MathReview

[10] S. Hirose, On diffeomorphisms over $T^{2}$-knot, Proc. of A.M.S. 119 (1993), 10091018. MathReview

[11] S. Hirose, On diffeomorphisms over trivially embedded in the 4-sphere, Algebr. Geom. Topol. 2 (2002) 791-824 MathReview

[12] L.K. Hua and I. Reiner, On the generators of the symplectic modular group, Trans. Amer. Math. Soc. 65 (1949), 415-426. MathReview

[13] Z. Iwase, Dehn surgery along a torus $T^{2}$-knot. II, Japan. J. Math. 16 (1990), 171-196. MathReview

[14] D. Johnson, The structure of the Torelli Group I: A finite set of generators for I , Ann. of Math. 118 (1983), 423-442. MathReview

[15] D. Johnson, The structure of the Torelli Group III: The abelianization of $\mathcal{I}$, Topology 24 (1985), 127-144. MathReview

[16] W.B.R. Lickorish, A finite set of generators for the homeotopy group of a 2-manifold, Proc. Cambridge Philos. Soc. 60 (1964), 769-778, Corrigendum: Proc. Cambridge Philos. Soc. 62 (1966), 679-681. MathReview

[17] Y. Matsumoto, An elementary proof of Rochlin's signature theorem and its extension by Guillou and Marin, A la Recherche de la Topologie Perdue, Progress in Math., 62 (1986), 119-139. MathReview 
[18] W. Magnus, A. Karras and D. Solitar, Combinatorial Group Theory, reprint of the 1976 second edition. Dover Publications, Inc. Mineola, NY (2004) MathReview

[19] J.M. Montesinos, On twins in the four-sphere I, Quart. J. Math. Oxford (2), 34 (1983), 171-199. MathReview

[20] V.R. Rohlin, Proof of a conjecture of Gudkov, Funkcional. Anal. i Priložen. 6 (1972) 62-64 (Russian); translation: Functional Analysis and its Applications, 6 (1972), 136-138. MathReview

[21] C.P. Rourke and D.P. Sullivan, On the Kervaire obstruction, Ann. of Math. (2) 94 (1971), 397-413. MathReview

Department of Mathematics, Faculty of Science and Engineering

Saga University, Saga, 840-8502 Japan.

Email: hirose@ms.saga-u.ac.jp

Received: 13 February 2005 Revised: 28 April 2005 